ARTICLE

Received 8 Nov 2013 | Accepted 7 Jan 2014 | Published 4 Feb 2014

DOI: $10.1038 /$ ncomms 4209

OPEN

\title{
Activation of the NLRP1b inflammasome independently of ASC-mediated caspase-1 autoproteolysis and speck formation
}

Nina Van Opdenbosch ${ }^{1,2}$, Prajwal Gurung ${ }^{3}$, Lieselotte Vande Walle ${ }^{1,2}$, Amelie Fossoul ${ }^{1,2}$, Thirumala-Devi Kanneganti ${ }^{3, \star} \&$ Mohamed Lamkanfi,2,*

Despite its clinical importance in infection and autoimmunity, the activation mechanisms of the NLRP1b inflammasome remain enigmatic. Here we show that deletion of the inflammasome adaptor ASC in BALB/c mice and in C57BL/6 macrophages expressing a functional NLRP1b prevents anthrax lethal toxin (LeTx)-induced caspase-1 autoproteolysis and speck formation. However, $\mathrm{ASC}^{-/-}$macrophages undergo normal LeTx-induced pyroptosis and secrete significant amounts of interleukin (IL)-1 $\beta$. In contrast, ASC is critical for caspase-1 autoproteolysis and IL-1 $\beta$ secretion by the NLRC4, NLRP3 and AIM2 inflammasomes. Notably, LeTx-induced inflammasome activation is associated with caspase-1 ubiquitination, which is unaffected in ASC-deficient cells. In vivo, ASC-deficient mice challenged with LeTx produce significant levels of IL-1 $\beta$, IL-18 and HMGB1 in circulation, although caspase-1 autoproteolysis is abolished. As a result, $\mathrm{ASC}^{-/-}$mice are sensitive to rapid LeTx-induced lethality. Together, these results demonstrate that ASC-driven caspase-1 autoprocessing and speck formation are dispensable for the activation of caspase-1 and the NLRP1b inflammasome.

\footnotetext{
${ }^{1}$ Department of Medical Protein Research, VIB, Ghent B-9000, Belgium. ${ }^{2}$ Department of Biochemistry, Ghent University, Ghent B-9000, Belgium.

${ }^{3}$ Department of Immunology, St Jude Children's Research Hospital, Memphis, Tennessee 38105-2794, USA. * These authors contributed equally to this work. Correspondence and requests for materials should be addressed to M.L. (email: mohamed.lamkanfi@vib-ugent.be).
} 
T he cysteine protease caspase- 1 plays a central role in proteolytically maturing and secreting the cytokines prointerleukin (IL)-1 $\beta$ and IL-18, and in inducing a proinflammatory programmed cell death mode termed pyroptosis ${ }^{1}$. In addition to IL-1 $\beta$ 's role as a critical pyrogen, both IL-1 $\beta$ and IL-18 shape innate and adaptive immune responses through a multitude of mechanisms that ultimately lead to coordinated host responses against infectious agents. In addition, recent reports highlighted a key role for caspase-1-mediated pyroptosis in controlling in vivo replication and dispersion of microbial pathogens ${ }^{2}$. Consequently, deregulated caspase-1 activation contributes importantly to a wide spectrum of infectious, autoimmune and inflammatory diseases ${ }^{3}$. To avoid unwarranted and potentially destructive immune responses, caspase- 1 activation in innate immune cells is regulated at several steps. Caspase- 1 is produced as a catalytically latent monomeric zymogen that resides in the cytosol of naive myeloid cells ${ }^{4}$. The zymogen consists of an amino-terminal caspase recruitment domain (CARD) that is separated from the catalytic protease domain by a short linker peptide. In addition, a short linker segregates the large (p20) and small (p10) subunits of the caspase proteolytic domain ${ }^{5}$. Imperative to gaining proteolytic activity, procaspase-1 needs to be recruited into cytosolic multi-protein complexes termed inflammasomes. Inflammasome complexes are assembled in a pathogen- or danger-associated molecular pattern (DAMP)-specific manner, and routinely distinguished based on the NOD-like receptor (NLR) or HIN200 pattern recognition receptor (PRR) facilitating caspase-1 autoactivation in the complex ${ }^{1}$.

Genetic studies in mice have confirmed the existence of at least four distinct inflammasomes responding to a diversity of PAMPs and DAMPs. The AIM2 inflammasome is assembled and activates caspase-1 when DNA is detected in the cytosolic compartment of macrophages infected with the DNA viruses cytomegalovirus and vaccinia virus, or the facultative intracellular bacterial pathogen Francisella tularensis, ${ }^{6,7}$. The NLRP3 inflammasome is required for caspase-1 activation and IL-1 $\beta /$ IL-18 secretion in response to a broad range of bacterial, viral and fungal pathogens, as well as medically relevant crystalline substances, and endogenous danger-associated molecular patterns that have been linked to the etiology of autoimmune and auto-inflammatory disorders ${ }^{1}$. The NLRC4 inflammasome responds to a more restricted set of bacterial components. This inflammasome was namely shown to be responsible for caspase-1 activation upon cytosolic detection of bacterial flagellin or the conserved PrgJ basal body of bacterial type III secretion systems expressed by facultative intracellular pathogens such as S. typhimurium, S. flexneri, $P$. aeruginosa, B. thailandensis, and L. pneumophila ${ }^{1}$. Finally, the NLRP1b inflammasome is critical for caspase-1 activation by anthrax lethal toxin (LeTx), a key virulence factor of Bacillus anthracis ${ }^{8}$.

Inhalation and ingestion of endospores of this Gram-positive rod-shaped bacterium is often fatal as a result of severe haemorrhagic disease. The spores are believed to target macrophages for their replication and systemic dispersion into the bloodstream 9 . Morbidity and mortality associated with systemic anthrax infection are caused by the tripartite anthrax toxin. The protective antigen (PA) subunit of the toxin binds to cell surface receptors of target cells and allows cytosolic entry of the metalloprotease lethal factor (LF) and the adenylate cyclase edema factor (EF) subunits. The combination of PA and EF (edema toxin) induces edema, whereas the combined action of PA and LF (called lethal toxin; LeTx) is responsible for lethal shock in experimental animals? ${ }^{9}$. The differential susceptibility of inbred mouse strains to LeTx-induced macrophage cell death and in vivo lethality was mapped to the gene encoding NLRP $1 \mathrm{~b}^{8}$. Cytosolic presence of LF triggers assembly of a caspase-1activating inflammasome complex in macrophages with a functional NLRP1b allele ${ }^{10}$. Recent studies showed that NLRP1b autoprocessing within the Function to Find Domain (FIIND) is required for LeTx-induced NLRP1b inflammasome activation ${ }^{11}$. Nevertheless, little is known about the molecular determinants and the mechanistic requirements leading to NLRP1b inflammasome-mediated caspase-1 activation, IL-1 $\beta$ secretion and cell death in LeTx-intoxicated macrophages and in in vivo-challenged animals. Particularly, the role of the inflammasome adaptor ASC in NLRP1b inflammasome signalling is debated due to the lack of appropriate ASC-deficient mouse models. Some reports relying on ectopic expression of inflammasome components suggested ASC to be dispensable for NLRP1b inflammasome signalling ${ }^{12,13}$, while other reports implicated ASC in NLRP1b inflammasome signalling ${ }^{14}$. Thus, the physiological role of ASC in NLRP1b inflammasome signalling remains to be fully addressed.

Here, we show in two genetic backgrounds that ASC is critical for NLRP1b inflammasome-mediated caspase-1 autoproteolysis and speck formation. Surprisingly, however, ASC is dispensable for LeTx-induced IL-1 $\beta$ secretion and pyroptotic cell death. Moreover, we find that activation of the NLRP1b inflammasome is associated with the ubiquitination of caspase-1, which requires NLRP1b and caspase-1 enzymatic activity, but not ASC. As in cultured macrophages, in vivo caspase-1 autoprocessing is blunted in splenocytes of ASC-deficient mice challenged with LeTx, while they produce significant levels of IL-1 $\beta$ and IL-18, and release the danger signal HMGB1 in circulation. Consequently, these mice succumb to LeTx intoxication with similar kinetics as $\mathrm{ASC}^{+/+}$littermates.

\section{Results}

ASC is critical for NLRP1b-mediated caspase-1 autoproteolysis. Recent reports showed that enzymatic activity of $\mathrm{LeTx}^{10}$ and a functional NLRP1b allele ${ }^{8}$ were critical for caspase-1 activation in intoxicated macrophages. In agreement, PA-mediated delivery of wild-type LF-but not the catalytically inactive $\mathrm{LF}_{\mathrm{E} 687 \mathrm{C}}$ mutant-potently triggered caspase-1 processing in bone marrow-derived macrophages (BMDMs) of $\mathrm{BALB} / \mathrm{c}$ mice (Fig. 1a). Unlike the BALB/c mouse strain, C57BL/6J macrophages express a dysfunctional NLRP1b allele, rendering them resistant to LeTx-induced caspase-1 activation ${ }^{8}$. Consequently, the combination of PA and LF failed to trigger caspase-1 processing (Fig. 1a) and membrane lysis in C57BL/6J macrophages (Fig. 1b). The bipartite inflammasome adaptor ASC plays a critical role in the NLRP3, AIM2 and NLRC4 inflammasomes ${ }^{15-18}$, but in-depth analysis of its role in NLRP1b inflammasome signalling was hampered by the LeTxresistant phenotype of $\mathrm{C} 57 \mathrm{BL} / 6 \mathrm{~J}$ mice. To characterize the role of ASC in NLRP1b inflammasome signalling, C57BL/6J (B6) mice transgenic for a functional NLRP1b allele transcribed from its endogenous promoter (referred to as $B 6^{N l p p I b+}$ mice) were bred to ASC-deficient mice. NLRP1b and ASC genotyping allowed segregation of $\mathrm{B}^{\mathrm{Nlrp} 16-}$ (further referred to as B6), B6 $6^{\mathrm{Nl} p 1 b+}$, $B 6^{\text {Nlrp } 1 b+} A_{S C} C^{+/-}$and $B 6^{\text {Nlrp } 1 b+} A S C^{-1-}$ littermates (Supplementary Fig. 1a), and RT-PCR analysis confirmed that transcripts levels of the functional NLRP1b allele in NLRP1btransgenic BMDMs were comparable to endogenous NLRP1b mRNA levels in 129SVEV57 macrophages (Supplementary Fig. 1b). Finally, western blot analysis confirmed intermediate ASC expression levels in $B 6^{\text {Nlrp } 1 b+} A S C^{+/-}$cells and absent expression in $B 6^{N l r p l b+} A S C^{-1-}$ macrophages (Supplementary Fig. 1c). In agreement with published results ${ }^{18,19}$, ASC plays a 
a

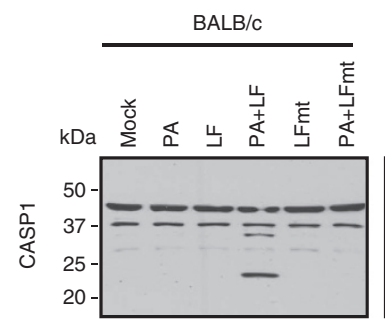

C

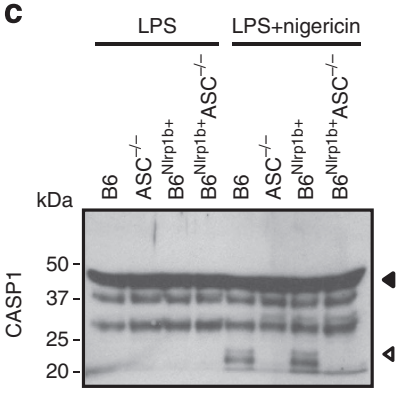

B6

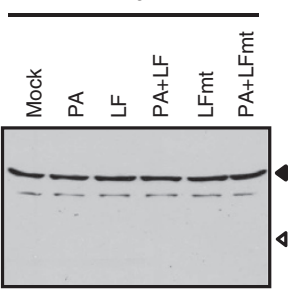

b

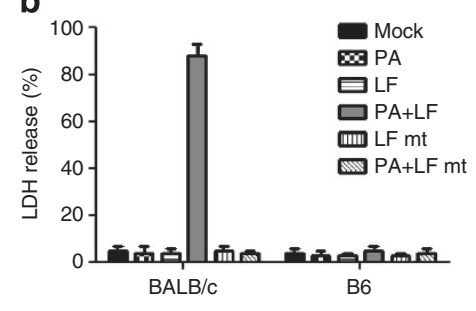

f

d

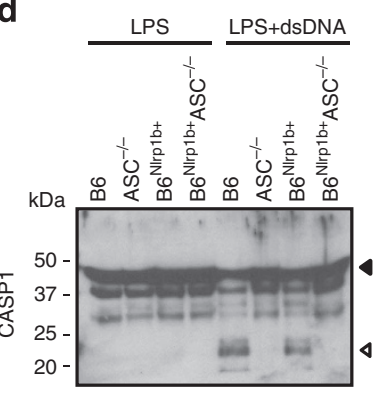

e

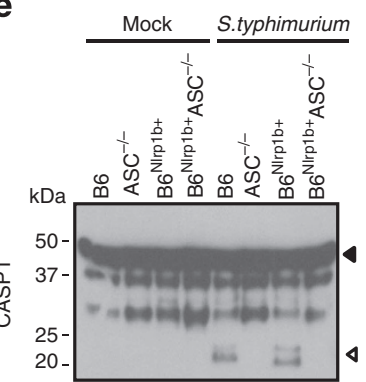

g LeTx (high)

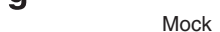

LeTx (low)
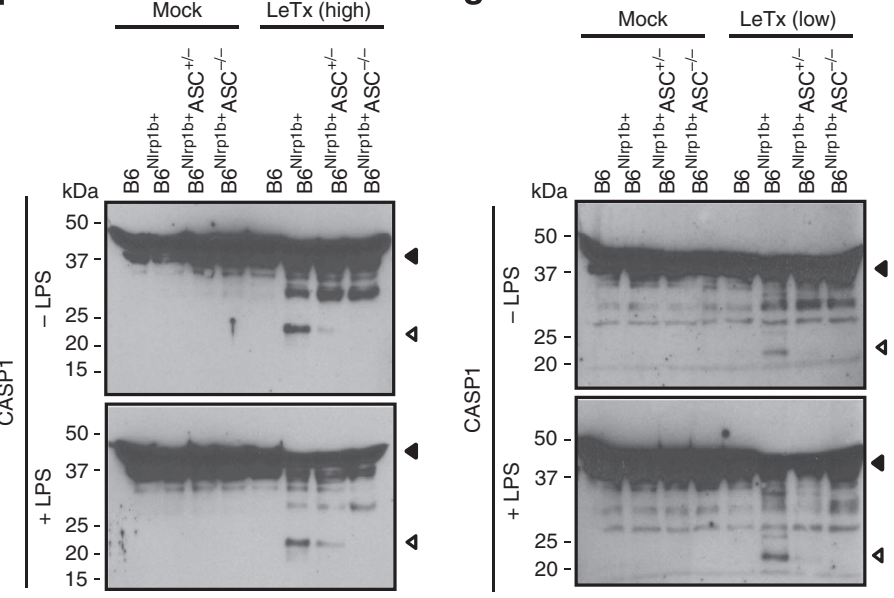

Figure 1 | ASC is critical for caspase-1 autoproteolysis by known inflammasomes. (a,b) BALB/C and B6 macrophages were treated with PA (10 $\mu g$ ) and/ or wild-type LF $(10 \mu \mathrm{g})$ and the enzymatically inactive $L_{\mathrm{E} 687 \mathrm{C}}$ mutant $(\mathrm{LFmt})$ for $3 \mathrm{~h}$, after which lysates were immunoblotted for caspase-1 (a) and culture supernatants analysed for $\mathrm{LDH}$ release (b). Data are shown as mean \pm s.d. from a single representative experiment of three experiments, with each condition performed in triplicate. (c-e) Lysates of $\mathrm{B} 6, \mathrm{ASC}^{-/-}, \mathrm{B}^{\mathrm{Nlrp} 1 \mathrm{~b}}+$ and $\mathrm{B}^{\mathrm{Nlrp} 1 \mathrm{~b}+} \mathrm{ASC}^{-/-} \mathrm{BMDMs}$ were immunoblotted for caspase-1 after cells have been primed with $5 \mu \mathrm{g} \mathrm{ml} \mathrm{m}^{-1}$ LPS for $3 \mathrm{~h}$ and subsequently stimulated with $20 \mu \mathrm{M}$ nigericin for $60 \mathrm{~min}$ (c), pretreated with $5 \mu \mathrm{g} \mathrm{ml}-1$ LPS ( $3 \mathrm{~h}$ ) and subsequently transfected with $1 \mu \mathrm{g}$ dsDNA for $24 \mathrm{~h}$ (d) or infected with S. typhimurium (m.o.i. 10) for $3 \mathrm{~h}(\mathbf{e})$. (f,g) B6, B $6^{\mathrm{Nlrp} 1 \mathrm{~b}+}+\mathrm{B}^{\mathrm{Nlrp} 1 \mathrm{~b}+}+\mathrm{ASC}+/-$ and $\mathrm{B}^{\mathrm{Nlrp} 1 \mathrm{~b}}+\mathrm{ASC}-/-\mathrm{BMDMs}$ were left untreated or pretreated with $5 \mu \mathrm{g} \mathrm{ml} \mathrm{I}^{-1} \mathrm{LPS}$ for $3 \mathrm{~h}$ prior to being exposed to different concentrations of LeTx (f, $10 \mu \mathrm{g}$ PA $+10 \mu \mathrm{g}$ LF) or ( $\mathbf{g}, 500 \mathrm{ng}$ PA $+250 \mathrm{ng}$ LF) for another $3 \mathrm{~h}$. Cell lysates were immunoblotted for caspase-1. Data are representative of results from three experiments.

critical role in caspase-1 autoproteolysis by the NLRP3 inflammasome because nigericin-induced caspase-1 autoprocessing into the p20 subunit was evident in LPS-primed B6 and $B 6^{\text {Nlrp } 1 b+}$ macrophages, but not in $A S C^{-1-}$ and $B 6^{\text {Nlrp } 1 b+} A S C^{-1-}$ cells (Fig. 1c). Similarly, ASC was necessary for caspase- 1 autoprocessing by respectively the AIM2 and NLRC4 inflammasomes because transfection of double-stranded DNA (dsDNA) and infection with Salmonella typhimurium (S. typhimurium) induced caspase-1 maturation in $\mathrm{B} 6$ and $B 6^{\text {Nlrp } 1 b+}$ macrophages, but not in ASC-deficient cells (Fig. 1d,e). To determine the role of ASC in caspase-1 autoproteolysis upon engagement of the NLRP1b inflammasome, macrophages of different genotypes were exposed to LeTx $\left(10 \mu \mathrm{g} \mathrm{ml}^{-1} \mathrm{PA}\right.$ and $\mathrm{LF}$, respectively) for $3 \mathrm{~h}$ before cell lysates were analysed for caspase-1 autoproteolysis. Unlike in B6 macrophages, LeTx potently induced caspase-1 autoproteolysis in $B 6^{N l r p i b+}$ macrophages that were or were not prestimulated with LPS (Fig. 1f). LeTx-induced caspase-1 processing was significantly reduced in macrophages from heterozygous $B 6^{\text {Nlrp } 1 b+} A S C^{+1-}$ littermates, and abolished in B6 $6^{\text {Nlrp } 1 b+}$ $A S C^{-1-}$ macrophages (Fig. 1f), suggesting a critical role for ASC in LeTx-induced caspase-1 autoproteolysis. Also, when exposed to a low concentration of LeTx $\left(500 \mathrm{ng} \mathrm{ml}^{-1} \mathrm{PA}\right.$ and $250 \mathrm{ng} \mathrm{ml}^{-1} \mathrm{LF}$, respectively), caspase-1 autoproteolysis was readily detectable in lysates of $B 6^{N l r p 1 b}+$ macrophages (Fig. 1g). In contrast, caspase-1 processing was markedly reduced in macrophages of $B 6^{\text {Nlrp } 1 b+} A S C^{+/-}$littermates, and fully absent in cells from $B 6^{\text {Nlrplb+}} A S C^{-1-}$ mice (Fig. 1g). Together, these results confirm that ASC is critical for caspase- 1 autoproteolysis by the NLRC4, NLRP3 and AIM2 inflammasomes, and reveal a 
similar requirement for ASC in caspase-1 autoprocessing by the NLRP1b inflammasome.

NLRP1b-driven pyroptosis is unaffected by ASC deletion. To understand the role of ASC in cell death induction, LPS-primed B6, $A S C^{-1-}, B 6^{N l r p 1 b+}$ and $B 6^{N l r p 1 b+} A S C^{-1-}$ macrophages
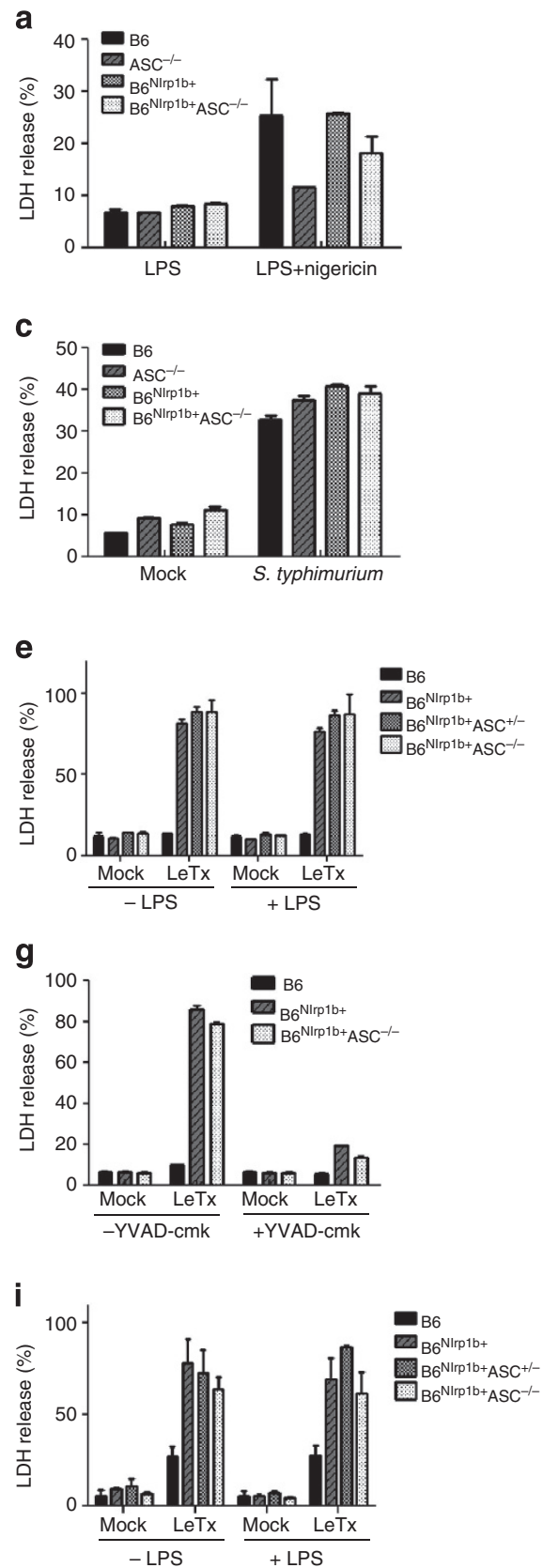

were stimulated with the NLRP3 inflammasome activator nigericin, or transfected with dsDNA to trigger the AIM2 inflammasome. Both nigericin and dsDNA transfection triggered cell death levels in B6 macrophages that were comparable to those of $B 6^{N l r p 1 b+}$ cells (Fig. 2a,b). Nigericin- and dsDNA-induced cell lysis was markedly-albeit not fully-inhibited in $A S C^{-/-}$and $B 6^{\text {Nlrp } 1 b+} A S C^{-1-}$ macrophages, suggesting that ASC is partially

b

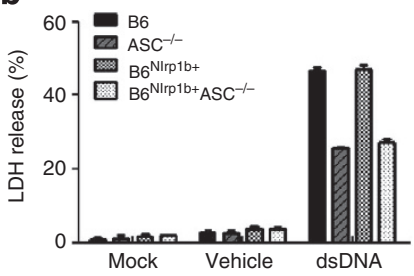

d
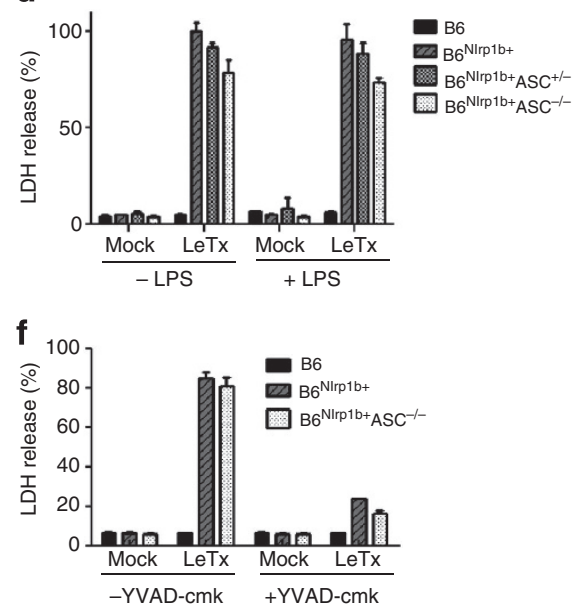

h
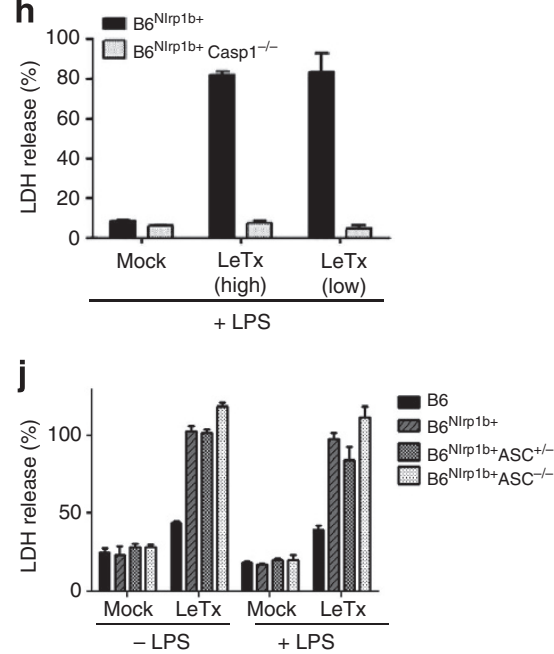

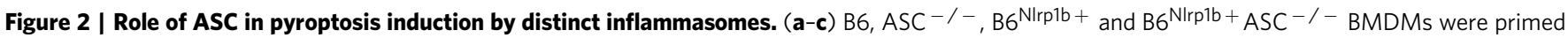
with $5 \mu \mathrm{g} \mathrm{ml}{ }^{-1}$ LPS for $3 \mathrm{~h}$ and subsequently stimulated with $20 \mu \mathrm{M}$ nigericin for 60 min (a) or transfected with $1 \mu \mathrm{g}$ dsDNA for $24 \mathrm{~h}$ (b), or infected with S. typhimurium (m.o.i. 10) for $3 \mathrm{~h}$ in the absence of LPS priming (c) before LDH levels were determined in culture supernatants. (d,e) B6, B6 ${ }^{\mathrm{Nlrp} 1 \mathrm{~b}+}$, $\mathrm{B}^{\mathrm{Nlrp} 1 \mathrm{~b}+} \mathrm{ASC} \mathrm{C}^{+/-}$and $\mathrm{B}^{\mathrm{Nlrp} 1 \mathrm{~b}+} \mathrm{ASC}-/-\mathrm{BMDMs}$ were left untreated or pretreated with $5 \mu \mathrm{g} \mathrm{ml} \mathrm{I}^{-1}$ LPS for $3 \mathrm{~h}$ prior to being exposed to different concentrations of LeTx (d, $10 \mu \mathrm{g}$ PA $+10 \mu \mathrm{g}$ LF) or (e, $500 \mathrm{ng}$ PA $+250 \mathrm{ng}$ LF) for another $3 \mathrm{~h}$ before LDH levels were determined in culture supernatants. $(\mathbf{f}, \mathbf{g})$ LPS-primed B6, B6 $6^{\mathrm{Nlrp} 1 \mathrm{~b}+}$ and B6 $6^{\mathrm{Nlrp} 1 \mathrm{~b}}+{ }_{\mathrm{ASC}}^{-/}-\mathrm{BMDMs}$ were pre-incubated with $50 \mu \mathrm{M}$ ac-YVAD-cmk for 30 min prior to LeTx treatment for $3 \mathrm{~h}$ $\left(\mathbf{f}, 10 \mu \mathrm{g}\right.$ PA $+10 \mu \mathrm{g} \mathrm{LF} ; \mathbf{g}, 500 \mathrm{ng}$ PA $+250 \mathrm{ng}$ LF) before LDH levels were determined in culture supernatants. (h) B6 $6^{\mathrm{Nlrp} 1 \mathrm{~b}+}$ and B6 ${ }^{\mathrm{Nlrp} 1 \mathrm{~b}+} \mathrm{Casp1}{ }^{-/}-$ BMDMs were primed with $5 \mu \mathrm{g} \mathrm{ml}^{-1}$ LPS for $3 \mathrm{~h}$ before LeTx treatment (high, $10 \mu \mathrm{g}$ PA $+10 \mu \mathrm{LF}$; low, $500 \mathrm{ng}$ PA $+250 \mathrm{ng}$ LF) for another $3 \mathrm{~h}$ followed by $\mathrm{LDH}$ measurement in culture supernatants. (i,j) B6, B6 $6^{\mathrm{Nlrp} 1 \mathrm{~b}}+, \mathrm{B}^{\mathrm{Nlrp} 1 \mathrm{~b}+} \mathrm{ASC}+/-$ and $\mathrm{B}^{\mathrm{N} N \mathrm{rp} 1 \mathrm{~b}+} \mathrm{ASC}-/-$ BMDMs were left untreated or primed with $5 \mu \mathrm{g} \mathrm{ml} \mathrm{m}^{-1} \mathrm{LPS}$ for $3 \mathrm{~h}$ before LeTx treatment (i, $10 \mu \mathrm{g}$ PA $+10 \mu \mathrm{g} \mathrm{LF} ; \mathbf{j}, 500 \mathrm{ng}$ PA $+250 \mathrm{ng}$ LF) for $24 \mathrm{~h}$ before LDH levels were determined in culture supernatants. Data are shown as mean \pm s.d. from a single representative experiment of three experiments, with each condition performed in triplicate. 
required for pyroptosis induction by the NLRP3 and AIM2 inflammasomes, respectively. As expected ${ }^{17,18}$, S. typhimurium infection induced similar pyroptosis levels in $\mathrm{B} 6$ and $A S C^{-1-}$ macrophages, as well as in $B 6^{N l r p 1 b+}$ and $B 6^{N l r p 1 b+} A S C^{-1-}$ cells (Fig. 2c), indicating that ASC is dispensable for NLRC4 inflammasome-mediated pyroptosis induction. ASC also is dispensable for pyroptosis induction by the NLRP1b inflammasome because cell death of macrophages exposed for $3 \mathrm{~h}$ to either high (Fig. 2d) or low (Fig. 2e) concentrations of LeTx was independent of ASC expression. Further titration of the LeTx concentration reduced cell death responses in ASC-expressing and ASC-deficient BMDMs to a similar extent, confirming that ASC is not required for LeTx-induced pyroptosis (Supplementary Fig. 2a). As expected, the caspase-1 inhibitor Ac-YVAD-cmk inhibited LeTx-induced caspase-1 autoproteolysis in B $6^{\text {Nlrp } 1 b+}$ cells (Supplementary Fig. 2b). Although caspase-1 was not processed in LeTx-treated $B 6^{N l r p 1 b+} A S C^{-1-}$ macrophages (Fig. 1f,g), these cells were equally sensitive to cell death blockade by Ac-YVAD-cmk as $B 6^{N l r p 1 b}+$ cells expressing ASC (Fig. 2f,g), suggesting that caspase-1 is active and induces pyroptosis in LeTx-treated macrophages irrespective of its processing status. Indeed, Ac-YVAD-cmk-mediated protection against pyroptosis was due to caspase- 1 inhibition because LeTxinduced pyroptosis was abrogated in $\mathrm{B6}^{\mathrm{Nlrp} 1 b+C a s p 1^{-1-}}$ macrophages (Fig. 2h). Notably, B6 macrophages (lacking a functional NLRP1b allele) that were exposed to LeTx for $3 \mathrm{~h}$ were resistant to pyroptosis induction (Fig. 2d,e), but extended incubation for $24 \mathrm{~h}$ triggered significant cell lysis with levels reaching about half of those seen in cells expressing a functional NLRP1b allele regardless of their ASC expression status (Fig. 2i,j). This suggests that macrophage cell death uniquely relies on the NLRP1b inflammasome during initial phases of LeTx intoxication, whereas sustained LeTx exposure triggers additional inflammasome-independent cell death mechanisms at later time points. Together, these results demonstrate that ASC is dispensable for early NLRP1b-mediated cell death induction in LeTx-intoxicated macrophages.

ASC is dispensable for lethal toxin-induced IL-1ß secretion. Our results showed that LeTx-induced pyroptosis was normal in ASC-deficient macrophages although caspase-1 autoproteolysis was abolished, implying that caspase-1 zymogen recruitment in the NLRP1b inflammasome is sufficient for gaining enzymatic activity in the absence of caspase-1 autoproteolysis. Apart from pyroptotic cell death, inflammasomes mediate secretion of mature IL- $1 \beta^{20}$. We therefore examined the role of ASC in IL-1 $\beta$ secretion by distinct inflammasomes. The NLRP3 inflammasome stimulus nigericin induced significant IL-1 $\beta$ secretion from LPS-primed B6 and $B 6^{N l r p 1 b+}$ macrophages, and these responses were abrogated in $A S C^{-1}$ and $B 6^{N r p 1 b+} A_{S C}-7-$ macrophages (Fig. 3a). Similarly, dsDNAmediated activation of the AIM2 inflammasome induced potent secretion of IL-1 $\beta$ in ASC-expressing macrophages, but not in cells lacking ASC (Fig. 3b). Also, NLRC4 inflammasome-mediated cytokine secretion by S. typhimuriuminfected macrophages required ASC (Fig. 3c). In sharp contrast, heterozygous and homozygous deletion of ASC failed to affect IL-1 $\beta$ secretion by LPS-primed $B 6^{\text {Nlrp } 1 b+}$ macrophages that have been treated with a high concentration of LeTx (Fig. 3d,e). As expected, B6 macrophages failed to secrete mature IL-1 $\beta$ into the culture medium, in agreement with the requirement for a functional NLRP1b allele. ASC deletion partially affected cytokine secretion from cells exposed to a lower concentration of LeTx for $3 \mathrm{~h}$, although $B 6^{\mathrm{Nlp} p 1 b+} A S C^{+/-}$and $B 6^{\mathrm{Nlrp} 1 b+}$ $A S C^{-1-}$ macrophages still secreted significant amounts of IL- $1 \beta$
(Fig. 3f,g). Similar results were obtained when macrophages were treated for $24 \mathrm{~h}$ : ASC-deficient cells secreted normal IL-1 $\beta$ levels when exposed to a high dose of LeTx, and reduced levels with a low LeTx concentration (Supplementary Fig. 3a,b). The inflammatory caspase inhibitor Ac-YVAD-cmk efficiently blocked LeTx-induced cytokine secretion (Fig. 3h,i), confirming that LeTx-induced IL-1 $\beta$ secretion required caspase-1. In agreement, genetic deletion of caspase-1 abrogated LeTxinduced IL-1 $\beta$ secretion from LPS-primed $\mathrm{B6}^{\mathrm{Nlrplb}+} \mathrm{Casp1^{-1- }}$ macrophages (Fig. 3j,k). A recent report showed that enteropathogen-induced caspase-1 autoproteolysis and IL-1 $\beta$ secretion by the non-canonical Nlrp3 inflammasome required caspase-11, and that $129 \mathrm{~S}$ macrophages are naturally deficient in caspase-11 expression $^{21}$. However, LeTx induced caspase-1 autoproteolysis (Fig. 31), IL-1 3 (Fig. 3m) and IL-18 (Fig. 3n) secretion in LPS-primed $129 \mathrm{~S}$ macrophages, indicating that caspase-11 is dispensable for LeTx-induced activation of the NLRP1b inflammasome. Together, these results indicate that, unlike its role in the NLRP3, AIM2 and NLRC4 inflammasomes, ASC is not critical for cytokine secretion by the NLRP1b inflammasome, although it enhances cytokine secretion at lower LeTx concentrations.

ASC and functional NLRP1b are essential for speck formation. ASC mediates the assembly of a single macromolecular perinuclear structure referred to as 'speck' in macrophages treated with stimuli of the NLRP3 (ref. 22), NLRC4 (ref. 17) and AIM2 inflammasomes ${ }^{23}$. These specks have been associated with efficient cytokine secretion by the NLRC4 inflammasome $e^{17}$, and cell death induction by the NLRP 3 inflammasome ${ }^{22}$. However, it is not known whether specks are formed under conditions of NLRP1b inflammasome activation. To examine LeTx-induced speck formation and its requirements, we analysed the subcellular localization of ASC and caspase-1 in B6, B6 $6^{\mathrm{Nlpp} 1 b+}$ and $B 6^{\text {Nlrp } 1 b+} A^{2} C^{-1-}$ macrophages by immunostaining before and after LeTx treatment. As expected, ASC mainly localized to the nuclear compartment of naive wild-type cells, and the immunofluorescent signal was largely lost in ASC cells (Supplementary Fig. 4a). Similarly, the caspase-1-specific antibody failed to stain caspase-1-deficient macrophages (Supplementary Fig. 4b), demonstrating specificity of the respective antibodies. LeTx treatment induced formation of a single, brightly stained perinuclear speck that contained both ASC and caspase- 1 in some B6 $6^{\text {Nlpplb }}+$ macrophages (Fig. 4a,b). Notably, these specks failed to form in LeTx-treated B6 and $B 6^{N l r p l b+}{ }^{2} C^{-C_{-}}$macrophages (Fig. 4a,b), demonstrating that a functional NLRP1b allele and ASC expression are essential for LeTx-induced speck formation.

To analyse whether these ASC specks contained active caspase-1, mock- and LeTx-treated macrophages were analysed by fluorescence microscopy using the active caspase-1 FLICA probe. The emission filter used to detect the FLICA signal passed a weak cellular autofluorescence background in mock-treated macrophages that was not due to the active caspase-1 probe because a similar background staining was evident in cells not exposed to the FLICA reagent (Supplementary Fig. 4c). In agreement with NLRP1b inflammasome-dependent caspase-1 activation, the FLICA signal increased significantly in $B 6^{N r p 1 b+}$ macrophages upon treatment with a high or low concentration of LeTx, but not in B6 macrophages lacking a functional NLRP1b allele (Fig. 4c). Notably, LeTx-treated $B 6^{\text {Nlrp } 1 b+}$ macrophages contained active caspase-1 in a single bright perinuclear speck and diffusely throughout the cell (Fig. 4c,d). Whereas diffuse active caspase-1 staining was retained in LeTx-treated $B 6^{\text {Nlrplb }} A S C^{-I-}$ macrophages, its focal 
localization in perinuclear specks was abrogated (Fig. 4c,d). Together, these results show that a functional NLRP1b allele and ASC expression are required for speck assembly and recruitment of active caspase-1 to these specks in LeTxintoxicated macrophages.
Role of ASC in lethal toxin-challenged BALB/c macrophages. Our results showed that ASC is required for caspase-1 autoprocessing and speck formation in LeTx-treated $B 6^{N l r p 1 b+}$ macrophages, but not for pyroptosis induction and IL-1 $\beta$ secretion. Although we assured that NLRP1b transcript levels in

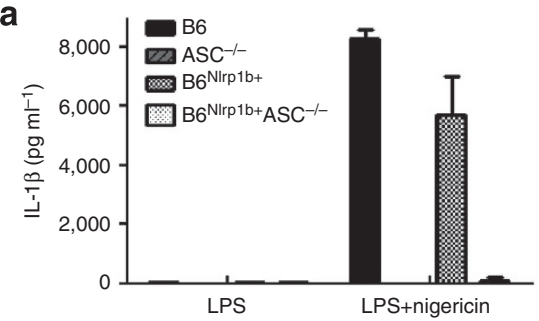

d

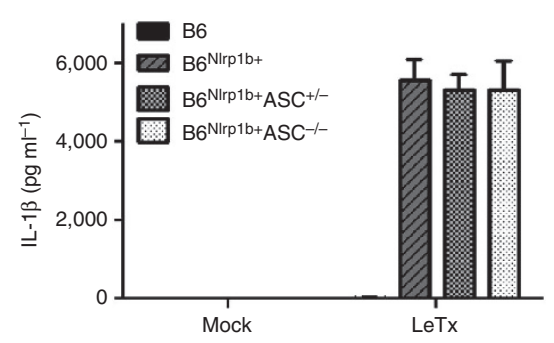

9

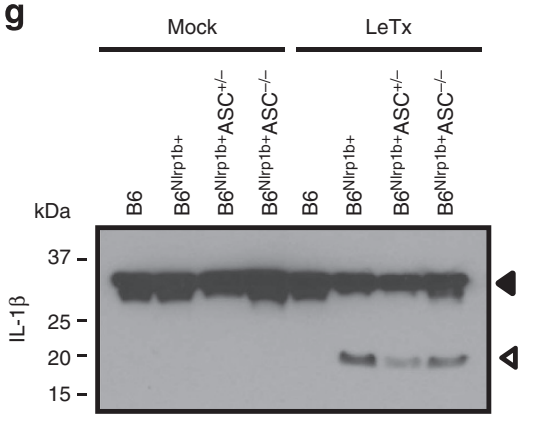

j

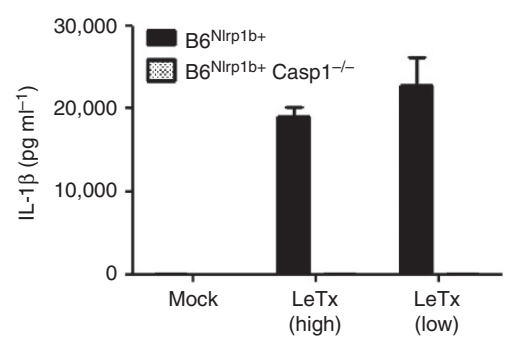

k

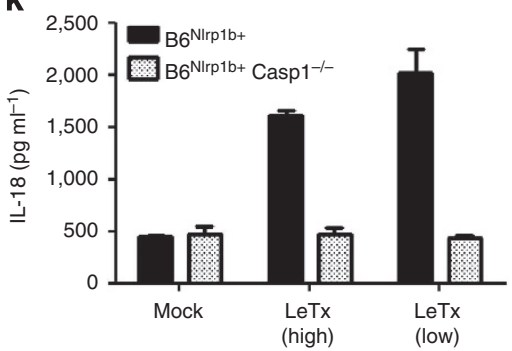

b

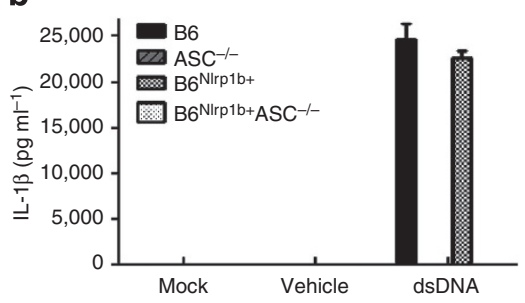

e

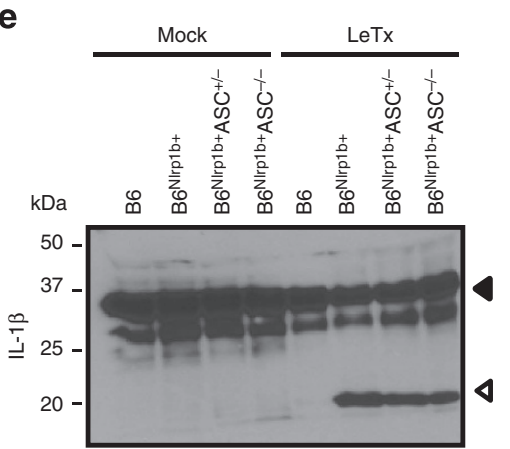

h

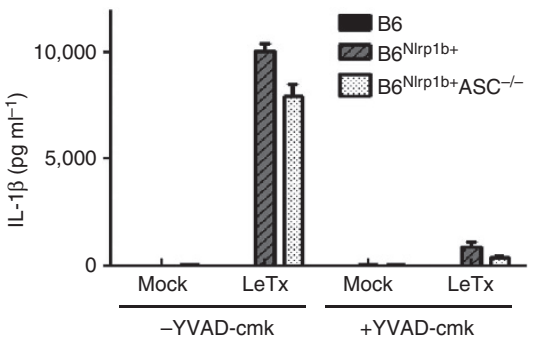

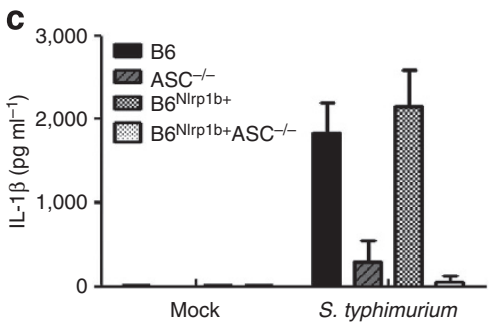

f

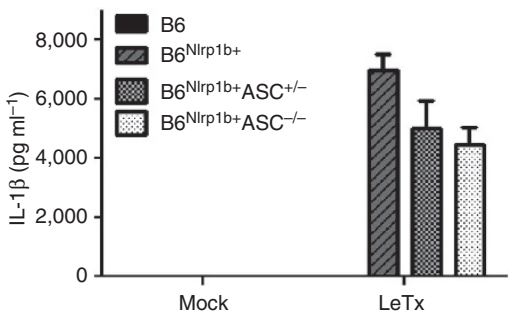

i

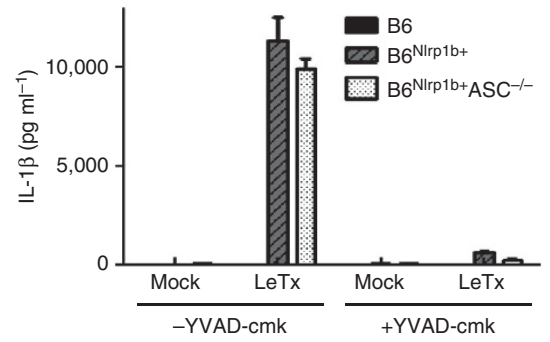

I

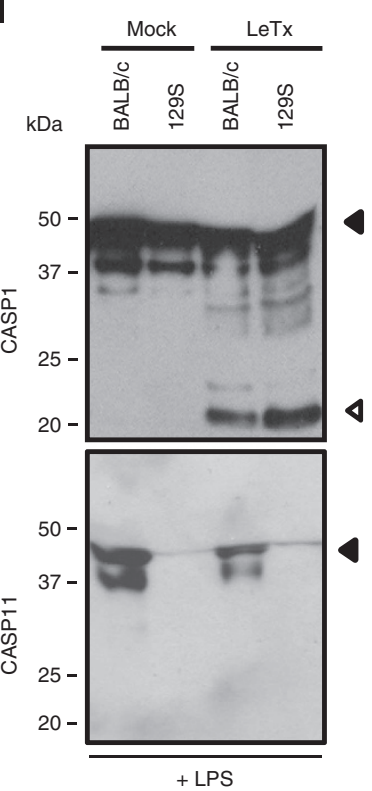

m

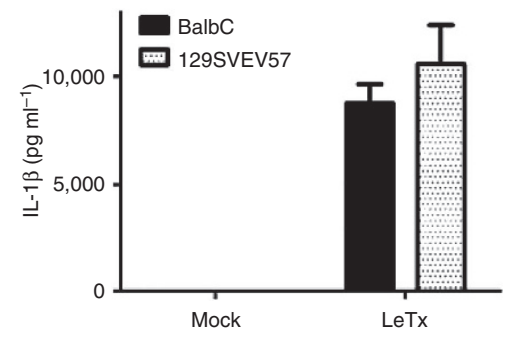

n

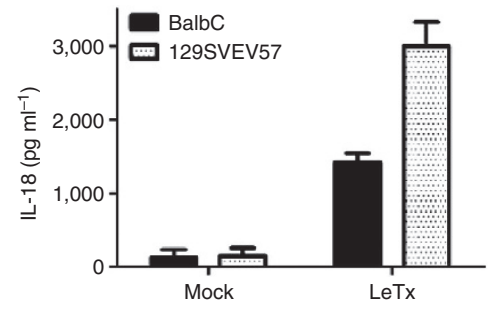


these cells were similar to those of endogenous NLRP1b in 129S macrophages (Supplementary Fig. 1b), we wanted to confirm our findings in the absence of transgenic NLRP1b expression. To this end, $A S C^{-1-}$ mice were bred to a $\mathrm{BALB} / \mathrm{c}$ genetic background for 10 generations before BMDMs from the resulting $A S C^{-1-}$ mice were intoxicated with LeTx. In agreement with our observations in $B 6^{N l r p 1 b+} A S C^{-1-}$ macrophages, lysates of BALB/c macrophages showed potent caspase-1 autoprocessing in response to LeTx treatment, and this was abrogated in the absence of ASC (Fig. 5a). As expected, specks were formed in LeTx-treated wildtype BALB/c macrophages, but not in cells lacking ASC (Supplementary Fig. 5). Despite defective caspase-1 autoproteolysis, pyroptosis induction in BALB/c $A S C^{-1-}$ macrophages treated with high or low concentrations of LeTx was similar to that of $\mathrm{ASC}$-expressing $\mathrm{BALB} / \mathrm{c}$ cells, respectively (Fig. 5b,c). Moreover, as we showed earlier for $B 6^{N l r p 1 b+}$ $A S C^{-1-}$ cells (Fig. $3 \mathrm{~d}$ ), secretion of IL- $1 \beta$ in response to high concentration of LeTx was similar in ASC-expressing and ASCdeficient $\mathrm{BALB} / \mathrm{c}$ macrophages (Fig. $5 \mathrm{~d}$,e), while cytokine secretion was partially affected in $A S C^{-9}$ cells when intoxicated with a lower concentration of LeTx (Fig. 5d,f). As in $B 6^{N l r p 1 b+}$ Casp $1^{-1-}$ macrophages, deletion of caspase-1 in BALB/c mice prevented LeTx-induced macrophage pyroptosis (Fig. $5 \mathrm{~g}$ ), and secretion of IL-1 $\beta$ and IL-18 (Fig. 5h,i). Together, these results confirm that LeTx induces pyroptosis and cytokine production in the absence of ASC-mediated caspase-1 autoprocessing, and that ASC specifically enhances cytokine production at lower LeTx concentrations.

Active caspase-1 is ubiquitinated independently of ASC. Our results suggest that NLRP1b and caspase-1 assemble a functional inflammasome independently of ASC. In agreement, caspase-1 was shown to specifically co-precipitate NLRP1b when ectopically expressed, suggesting that caspase- 1 and NLRP1b interact directly and that ASC is dispensable for this interaction ${ }^{13}$. Interestingly, caspase- 1 activation in $B 6^{\text {Nlrp } 1 b+}$ macrophages that were treated with either a high (Fig. 6a) or low (Fig. 6b) concentration of LeTx was consistently associated with the appearance of highmolecular weight species that are indicative of caspase-1 posttranslational modification. LeTx-induced caspase-1 modification required a functional NLRP1b allele, but was independent of LPS priming, ASC expression and caspase-1 autoproteolysis (Fig. 6a,b). The high-molecular weight bands represented modified caspase-1 species because LeTx-induced caspase-1 immunoreactive bands were apparent in lysates of intoxicated $B 6^{N l r p 1 b+}$ and BALB/c macrophages, but not in the corresponding LeTx-treated caspase-1-deficient cells (Fig. 6c,d). Stimulus-induced caspase-1 modification was not limited to the NLRP1b inflammasome because modified caspase-1 also was observed in LPS-primed macrophages that were treated with the NLRP3 stimuli ATP and nigericin (Fig. 6e), in B6 macrophages that were infected with Francisella tularensis to activate the AIM2 inflammasome (Fig. 6f), and in macrophages infected with different MOIs of the NLRC4 inflammasome-activating pathogen S. typhimurium (Fig. 6g). Inflammasome-induced caspase-1 modification required caspase- 1 enzymatic activity because AcYVAD-cmk inhibited LeTx-induced caspase-1 modification in both $B 6^{\text {Nlrplb+ }}$ (Fig. 6h) and $\mathrm{B6}^{\mathrm{Nl} p \mathrm{plb}+} \mathrm{ASC}^{-1-}$ (Fig. 6i) macrophages. We next immunoprecipitated caspase-1 from LeTx-challenged and LPS + ATP-treated macrophages to characterize the molecular identity of the caspase-1 posttranslational modification. These studies revealed caspase- 1 to be ubiquitinated under conditions promoting its activation by the NLRP1b and NLRP3 inflammasomes, respectively (Fig. 6j,k and Supplementary Fig. 6). In addition, we showed that LeTx-induced caspase-1 ubiquitination required NLRP1b and caspase-1 enzymatic activity, while ASC and caspase-1 autoproteolysis were dispensable.

In vivo role of ASC in the NLRP1b inflammasome. LeTx injection in vivo induces many symptoms associated with anthrax spore infection, including inflammation and death ${ }^{9}$. To address the physiological relevance of NLRP1b inflammasome signalling in the absence of ASC-dependent caspase-1 autoproteolysis, groups of $\mathrm{B} 6, B 6^{\mathrm{Nlp} p 1 b+}$ and $B 6^{\mathrm{Nl} p \mathrm{lb}+} \mathrm{ASC} \mathrm{C}^{-1-}$ mice were injected intraperitoneally with LeTx $\left(100 \mu \mathrm{gg}^{-1} \mathrm{PA}\right.$ and LF, respectively). Western blot analysis of whole spleen lysates prepared $3 \mathrm{~h}$ post injection showed that LeTx efficiently induced caspase-1 autoprocessing in $B 6^{\text {Nlrp } 1 b+}$ mice, but not in B6 mice (Fig. 7a). As in macrophages, these results demonstrate that a functional NLRP1b allele is critical for caspase-1 autoprocessing in LeTx-induced animals. Caspase-1 cleavage also was abrogated in $B 6^{N l r p l b+}$ mice deficient in ASC expression (Fig. 7a), confirming the critical role of ASC in LeTx-induced caspase-1 autoproteolysis in vivo. To study the in vivo role of NLRP1b and ASC in LeTx-induced cell death, serum was analysed for release of HMGB1, a danger signal released by dying cells during trauma and infection ${ }^{20}$. Concurrent with our observation that LeTx-induced macrophage cell death in a NLRP1b-dependent and ASC-independent manner, LeTxinduced levels of circulating HMGB1 were similarly elevated in $B 6^{\text {Nlrplb }}+$ and $B 6^{\text {Nlrplb+}} A S C^{-1-}$ mice, whereas levels of circulating HMGB1 in serum samples of unchallenged $B 6^{N l r p 1 b+}$ and $B 6^{N l p p 1 b+} A S C^{-1-}$ mice and LeTx-challenged B6 mice were below the detection limit of the antibody (Fig. 7b). LeTx-induced secretion of IL-1 $\beta$ (Fig. 7c) and IL-18 (Fig. 7d) also required a functional NLRP1b allele because B6 mice failed to release these inflammasome-dependent cytokines when challenged with LeTx. Notably, $B 6^{N l r p l b+} A S C^{-1}-$ mice contained high levels of circulating IL-1 $\beta$ and IL-18, although these were statistically lower than those of $B 6^{N l r p l b+}$ littermates (Fig. $7 \mathrm{c}, \mathrm{d}$ ). These results confirm that ASC is not essential but contributes to

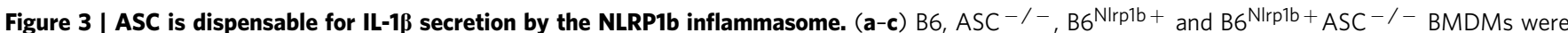
primed with $5 \mu \mathrm{g} \mathrm{ml}^{-1}$ LPS for $3 \mathrm{~h}$ and subsequently stimulated with $20 \mu \mathrm{M}$ nigericin for $60 \mathrm{~min}$ (a) or transfected with $1 \mu \mathrm{g}$ dsDNA for $24 \mathrm{~h}$ (b) or infected with S. typhimurium (m.o.i. 10) for $3 \mathrm{~h}$ in the absence of LPS priming (c) before secreted IL-1 $\beta$ levels were determined in culture supernatants. (d-g) B6,

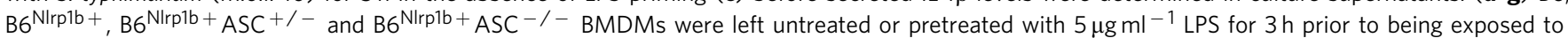
different concentrations of LeTx (d,e, $10 \mu \mathrm{g}$ PA $+10 \mu \mathrm{g}$ LF) or ( $\mathbf{f}, \mathbf{g}, 500 \mathrm{ng}$ PA $+250 \mathrm{ng}$ LF) for another $3 \mathrm{~h}$. Secreted IL- $1 \beta$ levels were determined in culture supernatants by ELISA and lysates were immunoblotted for IL-1 $\beta$. (h,i) LPS-primed B6, B6 $6^{\mathrm{N} / r p 1 b}+$ and B6 ${ }^{\mathrm{Nlrp} 1 \mathrm{~b}+}{ }^{\mathrm{ASC}}{ }^{-/-} \mathrm{BMDMs}$ were pre-incubated with $50 \mu \mathrm{M}$ ac-YVAD-cmk for 30 min prior to LeTx treatment for $3 \mathrm{~h}(\mathbf{h}, 10 \mu \mathrm{g}$ PA $+10 \mu \mathrm{g}$ LF; $\mathbf{i}, 500 \mathrm{ng}$ PA $+250 \mathrm{ng} \mathrm{LF}$ ). Secreted IL-1 13 levels were determined in culture supernatants. (j,k) B6 $6^{\text {Nlrplb }}+$ and B6 $6^{\mathrm{Nlrp1b}}+\mathrm{Casp1}^{-/-}$BMDMs were primed with $5 \mu \mathrm{g} \mathrm{ml} \mathrm{I}^{-1} \mathrm{LPS}$ for $3 \mathrm{~h}$ followed by LeTx treatment (high, $10 \mu \mathrm{g}$ PA $+10 \mu \mathrm{g} \mathrm{LF}$; low, $500 \mathrm{ng}$ PA $+250 \mathrm{ng}$ LF) for another $3 \mathrm{~h}$. Levels of secreted IL-1 $\mathbf{3}$ (j) and IL-18 (k) were determined in culture supernatants. (I-n) BALB/C and 129SVEV57 BMDMs were primed with $5 \mu \mathrm{g} \mathrm{ml}{ }^{-1}$ LPS for $3 \mathrm{~h}$ prior to LeTx treatment $(10 \mu \mathrm{g}$ PA $+10 \mu \mathrm{L}$ LF) for another $3 \mathrm{~h}$. Lysates were immunoblotted for the indicated proteins (I) and secreted IL-1 $\beta$ and IL-18 were determined in culture supernatants by ELISA (m,n). Data are shown as mean \pm s.d. from a single representative experiment of three experiments, with each condition performed in triplicate. 
a
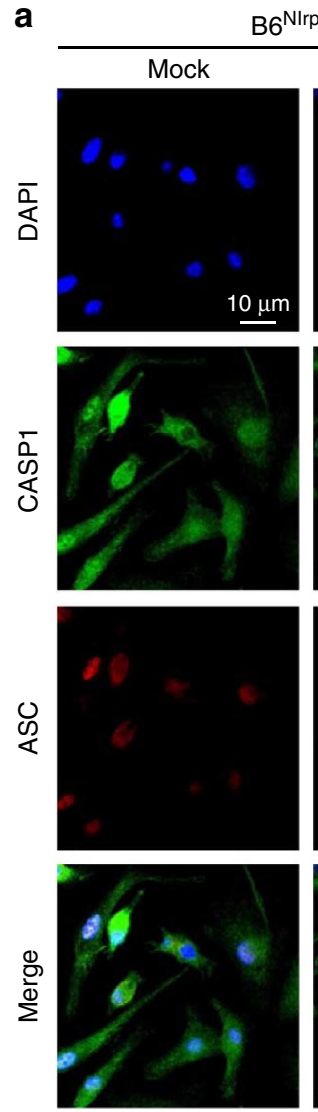

b
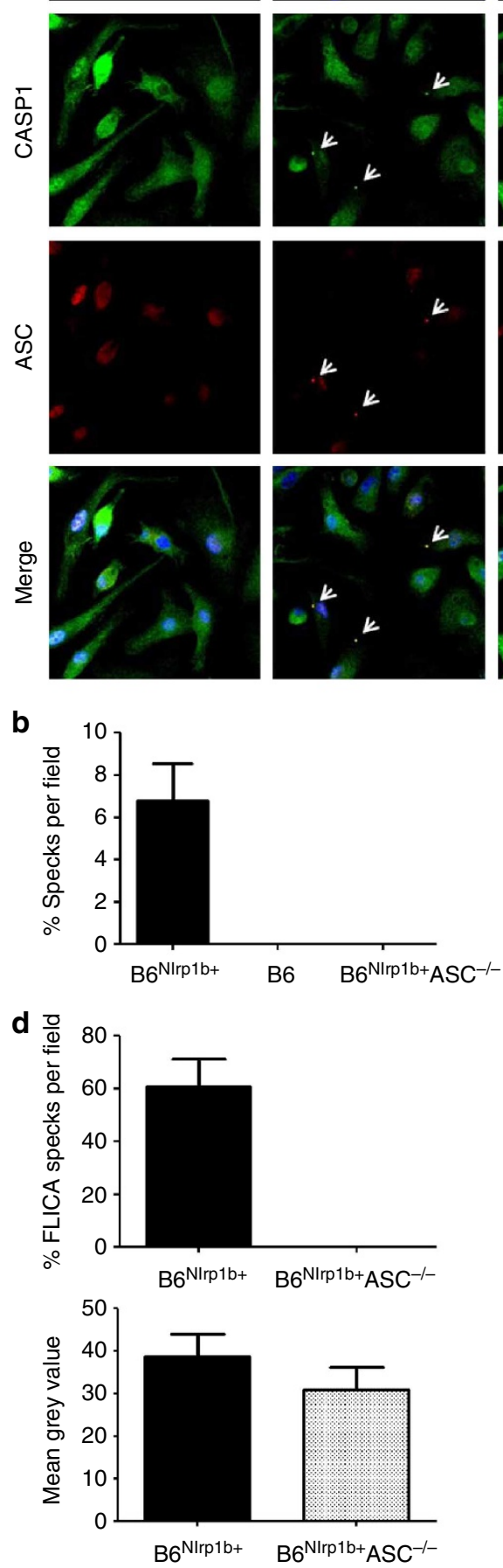

B6
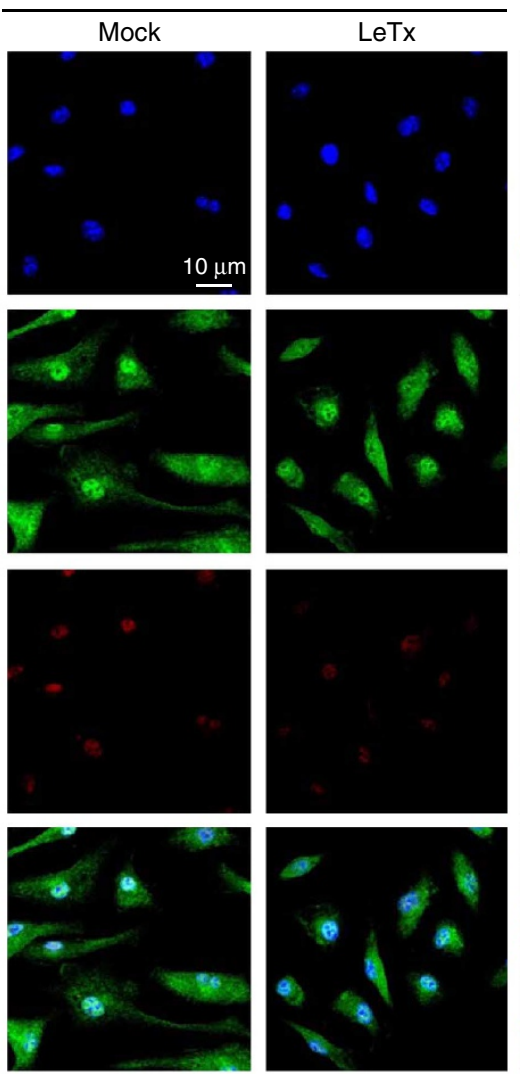

C
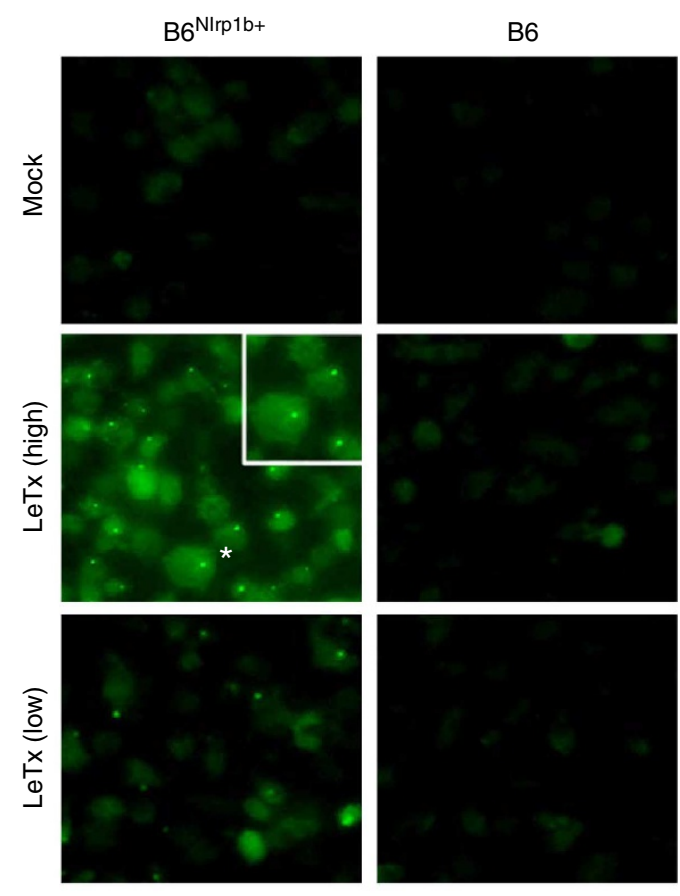
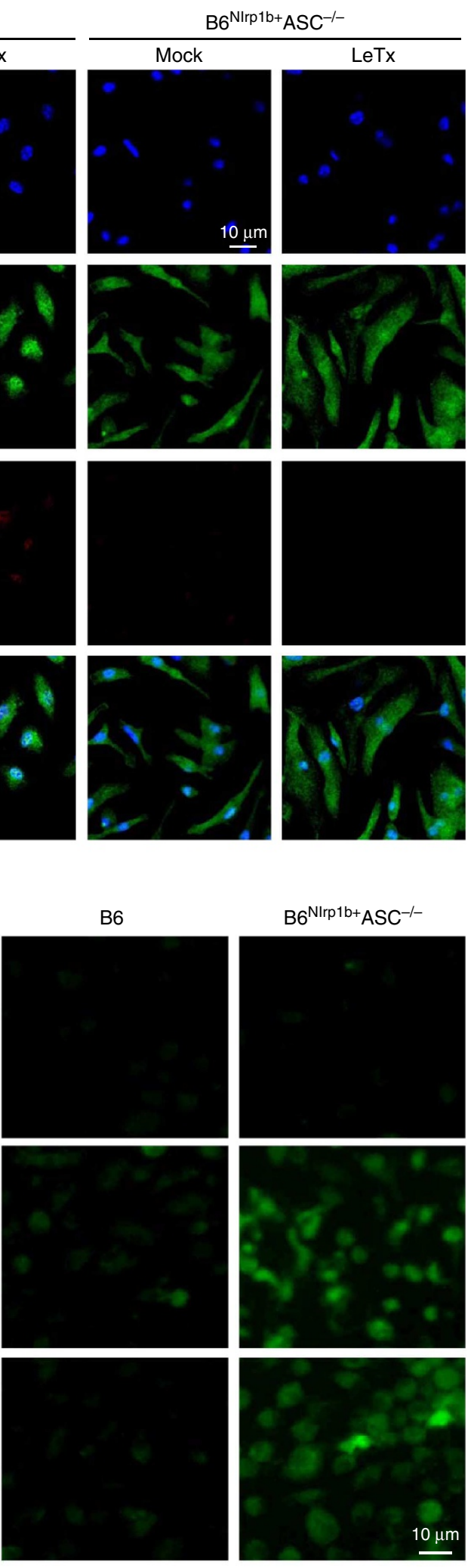

$\mathrm{B}^{\mathrm{NIrp} 1 \mathrm{~b}+} \mathrm{ASC}^{-/-}$
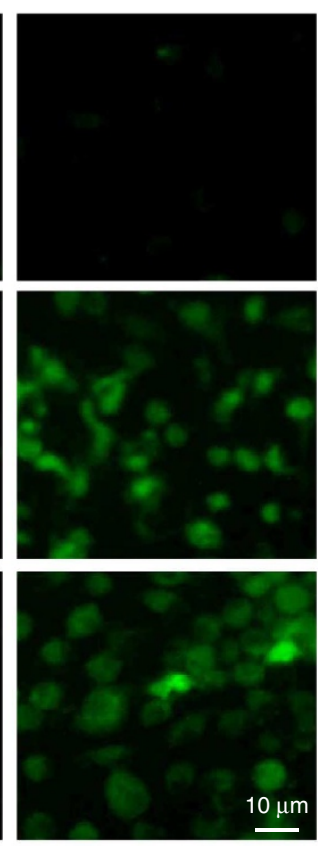

Figure 4 | ASC is critical for LeTx-induced speck formation. (a) B6, B6 $6^{\mathrm{Nlrp} 1 \mathrm{~b}}+$ and $\mathrm{B}^{\mathrm{N} 1 \mathrm{rp} 1 \mathrm{~b}}+\mathrm{ASC}-/-$ BMDMs were grown on coverslips and treated with LeTx (10 $\mu$ g PA and LF, respectively) for 60 min after which cells were fixed in 4\% paraformaldehyde and immunostained for ASC (red) and caspase-1 (green). DAPI (blue) was included to localize nuclei. Arrows indicate specks. Confocal micrographs were acquired at $\times 60$ magnification.

(b) Quantification of specks per field. (c) B6, B6 $6^{\mathrm{Nlrp} 1 \mathrm{~b}+}$ and $\mathrm{B}^{\mathrm{Nlrp} 1 b}+\mathrm{ASC}-/-\mathrm{BMDMs}$ that were stimulated with LeTx (high, $10 \mu \mathrm{g}$ PA $+10 \mu \mathrm{g}$ LF; low, $500 \mathrm{ng}$ PA $+250 \mathrm{ng}$ LF) for $30 \mathrm{~min}$, and then incubated with the active caspase-1 Fam-YVAD-fmk FLICA probe for 45 min before fluorescent micrographs were acquired at $\times 20$ magnification. ${ }^{*}$ indicates magnified area. (d) Quantification of FLICA-positive cells/field and mean grey value. Data are representative of results from two independent experiments and quantification data are presented as mean \pm s.d. from a single representative experiment. 
NLRP1b-mediated cytokine secretion in vivo. To address the importance of NLRP1b- and ASC-mediated signalling for LeTxinduced lethality, cohorts of mice were challenged with a lethal dose of LeTx (200 $\mathrm{gg} \mathrm{g}^{-1} \mathrm{PA}$ and LF, respectively). In agreement with published reports suggesting that LeTx susceptibility of inbred mouse and rat strains correlated with the rodent chromosomal loci encoding NLRP1 $b^{24,25}$, LeTx-challenged B6 mice resisted significantly longer to LeTx-induced death than B6 mice expressing a functional NLRP1b allele (Fig. 7e). Similarly, $B 6^{\text {Nlrplb }}+$ ASC $^{-1-}$ mice succumbed much faster to LeTx challenge than B6 mice, although they died with similar kinetics as $B 6^{\text {Nlrp } 1 b+}$ littermates (Fig. 7e). Together, these findings suggest a mechanistic model in which functional NLRP1b directly (independently of ASC) recruits procaspase-1 zymogens to assemble octa- or heptameric NLRP1b inflammasomes upon detection of LF metalloprotease activity in the cytosol (Fig. 7f). Procaspase-1 is likely recruited to these complexes by means of direct homotypic interactions between its prodomain and the C-terminal CARD of NLRP1b. Inflammasome complex assembly may induce conformational changes in procaspase-1 that allow it to gain enzymatic activity and cleave IL-1 $\beta$, IL-18 and yet unknown substrates leading to cell death. ASC-mediated oligomerization of multiple (ASC-free) NLRP1b inflammasomes induces formation of a single, large perinuclear speck in which procaspase-1 undergoes autoproteolysis, which may serve to stabilize the active protease and further enhance cytokine production (Fig. 7f).

\section{Discussion}

LeTx is lethal to nearly all mammalian species, and is considered the major virulence factor of $B$. anthracis because in vivo LeTx intoxication mimics disease progression in mammalian hosts that have been infected with $B$. anthracis spores, the causative agent of anthrax ${ }^{9,26}$. Depending on the route of infection, resident macrophages of respectively the lung, intestine or skin are targeted by $B$. anthracis spores for germination and bacterial replication, which may result in systemic dispersion of the infectious agent into the bloodstream ${ }^{27,28}$. Macrophages from inbred mouse strain respond differentially to LeTx intoxication. Those that express a functional NLRP1b allele (such as $129 \mathrm{~S}$ and $\mathrm{BALB} / \mathrm{c}$ ) induce rapid cell lysis, whereas those of resistant mouse strains (such as C57BL/6) encode mutated NLRP1b alleles ${ }^{8}$. Moreover, NLRP1b-induced macrophage cell death is-at least partially-correlated with the relative susceptibility of inbred mouse and rat strains to rapid LeTx-induced mortality in vivo ${ }^{24,25,28}$.

Upon PA-mediated access to the cytosolic compartment of intoxicated macrophages, the LF zinc protease subunit of LeTx cleaves MKK proteins and currently unknown substrates ${ }^{29,30}$. The host's immune system responds to LeTx proteolytic activity in the cytosol with assembly of the NLRP1b inflammasome, a protein complex that facilitates activation of the inflammatory cysteine protease caspase-1 (ref. 1). NLRP1b-activated caspase-1 converts the inflammatory cytokines IL-1 $\beta$ and IL-18 into their secreted bioactive forms, and induces pyroptotic cell death in sensitive macrophages ${ }^{20}$. However, the molecular composition and mechanisms by which the NLRP1b inflammasome controls caspase-1 activation, cytokine secretion and pyroptosis are unclear. Some reports relying on ectopic expression of inflammasome components suggested ASC to be dispensable for NLRP1b inflammasome signalling ${ }^{12,13}$, while other reports implicated ASC in NLRP1b inflammasome signalling ${ }^{14}$. Our analysis of LeTx-sensitive ASC-sufficient and -deficient cells and mice revealed that ASC was critical for particular, but not all, aspects of NLRP1b inflammasome signalling. Notably, co- incubation of recombinant human procaspase-1 and NLRP1 in vitro in the absence of ASC elicited caspase- 1 autoproteolysis ${ }^{31}$, whereas our studies in ASC-deficient macrophages indicated ASC to be required for caspase-1 autoprocessing by the NLRP1b inflammasome. Moreover, using fluorescence and confocal microscopic analysis, we demonstrated that speck formation occurs in LeTx-treated macrophages, and that their assembly was strictly dependent on the expression of ASC and a functional NLRP1b allele. In addition, we showed that these specks correlated with focal localization of active caspase-1 in the perinuclear region of intoxicated wild-type cells. However, despite absent speck formation, ASC-deficient macrophages secreted significant amounts of IL-1 $\beta$. In addition, ASC-deficient macrophages underwent normal LeTx-induced cell lysis. In contrast, both IL-1 $\beta$ secretion and pyroptosis were ablated in cells lacking a functional NLRP1b allele, or when caspase-1 activity was pharmacologically inhibited, confirming that these responses were dependent on a functional NLRP1b inflammasome. Given that ASC was critical for cytokine production by the latter complexes, these observations suggest that the NLRP1b inflammasome differs fundamentally from other known (NLRP3, NLRC4 and AIM2) inflammasomes. They also suggest that the functional NLRP1b inflammasome is a structure distinct from ASC specks because NLRP1b- and caspase-1dependent cytokine secretion and pyroptosis occurred in the absence of the latter. Notably, despite significant induction of cell death and cytokine secretion, ASC-deficient macrophages were fully defective in LeTx-induced caspase-1 autoproteolysis. This suggests that the absence of caspase- 1 autoproteolysis-a parameter widely used to monitor inflammasome activationalone is not sufficient to rule out inflammasome activation. In this regard, the general mechanisms of caspase-1 activation in inflammasome complexes may much resemble those governing activation of the apoptotic initiator caspases 8 and -9 in the death-inducing signalling complexes and the apoptosome, respectively ${ }^{32,33}$. We therefore propose a model in which NLRP1b directly recruits caspase-1 via homotypic CARDCARD interactions to assemble a functional inflammasome complex in the cytosol of LeTx-sensitive macrophages. Unprocessed, but enzymatically active caspase-1 in the NLRP1b inflammasome triggers cytokine production and pyroptotic cell death. ASC-mediated clustering of multiple NLRP1b inflammasomes in specks further increases the local concentration of active caspase-1 dimers, promoting their proximity-induced autoproteolysis. While this may generally serve to stabilize the active protease, further analysis is required to understand why ASC specifically enhanced IL- $1 \beta$ secretion, but not cell lysis of macrophages exposed to low LeTx concentrations.

Notably, caspase-1 activation in the NLRP1b inflammasome was associated with its ubiquitination, which occurred independently of ASC expression and caspase-1 autoproteolysis. Interestingly, caspase-1 ubiquitination was not limited to the NLRP1b inflammasome as it was also observed upon activation of the NLRP3, AIM2 and NLRC4 inflammasomes. Caspase-1 ubiquitination likely occurs downstream of caspase-1 activation in inflammasomes because the caspase-1 inhibitor Ac-YVAD-cmk prevented LeTx-induced caspase-1 post-translational modification. We therefore hypothesized that caspase-1 ubiquitination may represent a negative feedback mechanism that dampens inflammasome activation by targeting active caspase- 1 for degradation by the proteasome. However, the proteasome inhibitor MG132 inhibited LeTx-induced pyroptosis and caspase-1 autoproteolysis altogether (data not shown), thus hampering investigation of the potential role of the proteasome in degrading inflammasome-activated caspase- 1 . However, future identification of the molecular machinery driving caspase-1 
a

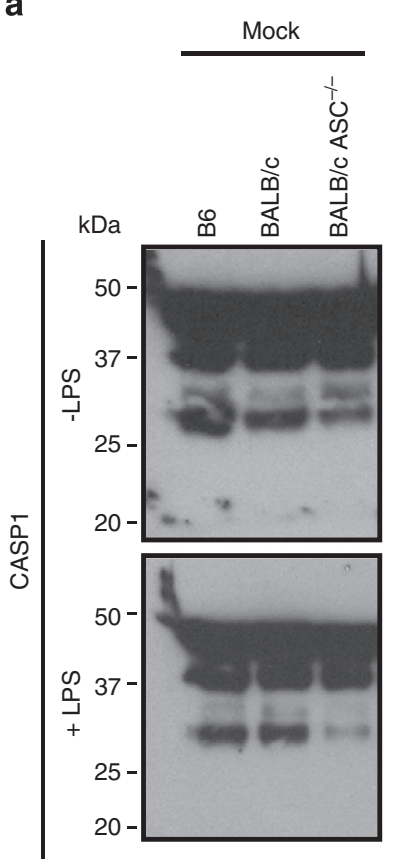

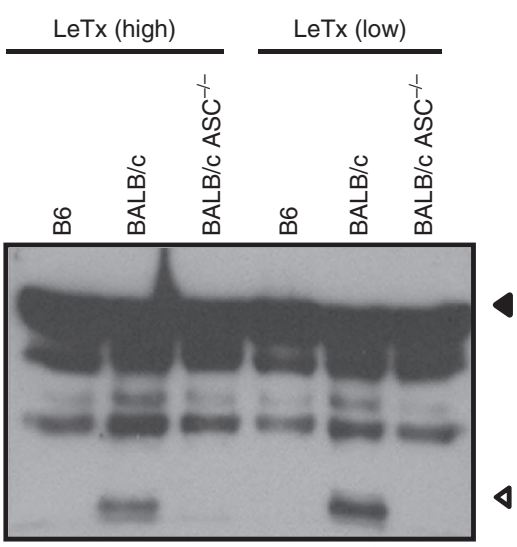

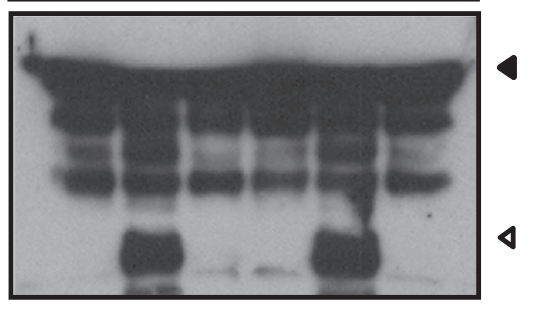

e b

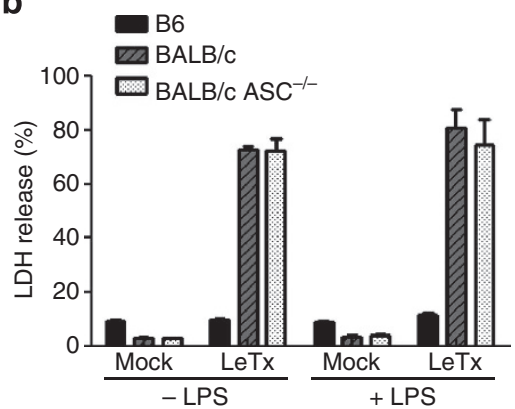

C

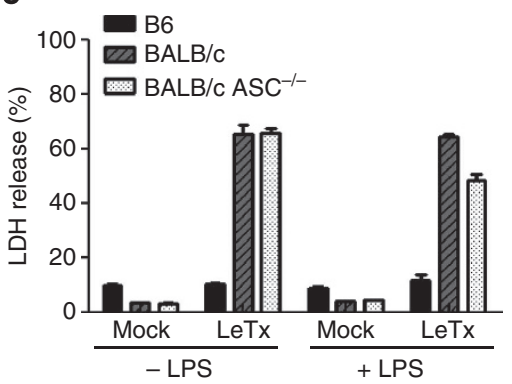

d

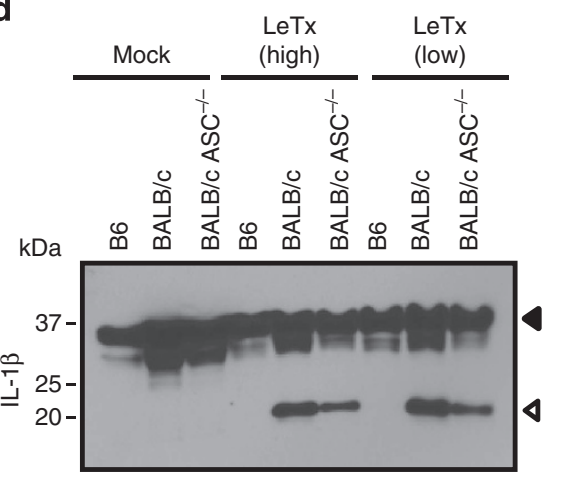

g

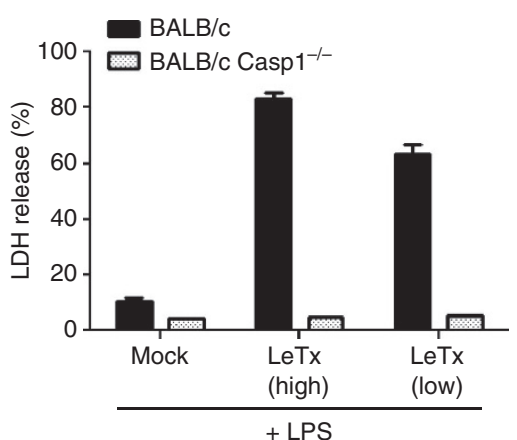

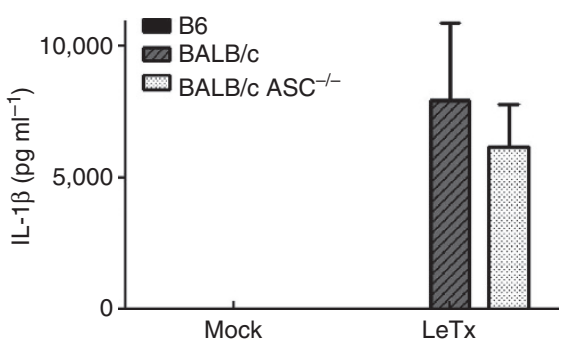

h

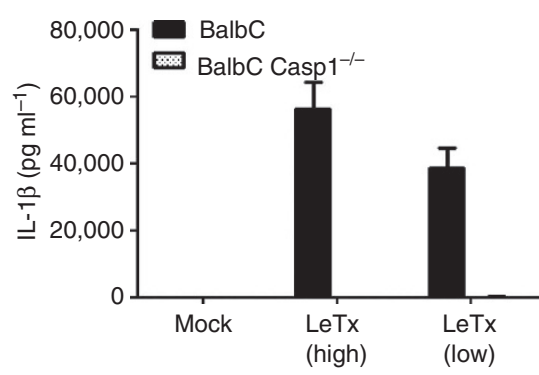

f

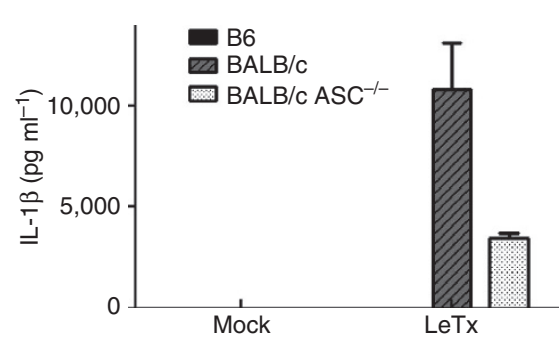

i

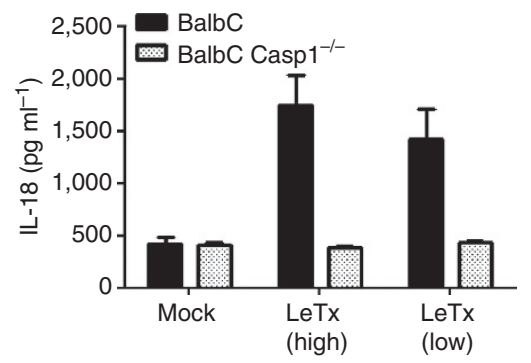

Figure 5 | ASC-deficiency in BALB/c macrophages prevents LeTx-induced caspase-1 autoproteolysis but not pyroptosis and IL-1 secretion. (a-f) B6, $\mathrm{BALB} / \mathrm{c}$ and $\mathrm{BALB} / \mathrm{C} \mathrm{ASC}-1-\mathrm{BMDMs}$ were left untreated or pretreated with $5 \mu \mathrm{g} \mathrm{ml} \mathrm{m}^{-1} \mathrm{LPS}$ for $3 \mathrm{~h}$ before incubation with LeTx (high, $10 \mu \mathrm{g}$ PA $+10 \mu \mathrm{g}$ LF; low, $500 \mathrm{ng}$ PA $+250 \mathrm{ng}$ LF) for another $3 \mathrm{~h}$. Cell lysates were immunoblotted for caspase-1 and IL-1 $\beta$ (a,d) and culture supernatants were analysed for $\operatorname{LDH}(\mathbf{b}, \mathbf{c})$ and secreted IL-1 $\beta(\mathbf{e}, \mathbf{f})$. (g-i) BALB/c and BALB/c Casp1 ${ }^{-/-}$BMDMs were primed with $5 \mu \mathrm{g} \mathrm{ml}{ }^{-1}$ LPS for $3 \mathrm{~h}$ followed by LeTx treatment (high, $10 \mu \mathrm{g} \mathrm{PA}+10 \mu \mathrm{LF}$; low, $500 \mathrm{ng}$ PA $+250 \mathrm{ng}$ LF) for another $3 \mathrm{~h}$. Levels of LDH (g) and secreted IL-1 3 (h) and IL-18 (i) were determined in culture supernatants. Data are representative of results from three experiments, and cytokine and LDH data are presented as mean \pm s.d. from a single representative experiment, with each condition performed in triplicate.

ubiquitination may shed light on its putative role(s) in inflammasome signalling.

Regardless, we showed that the differential requirement of ASC for caspase-1 autoproteolysis and its biological effects also applies to mice challenged in vivo with LeTx. As in macrophages,
LeTx-induced caspase- 1 autoproteolysis in the spleen required ASC and a functional NLRP1b allele. Nevertheless, LeTxchallenged ASC-deficient mice released HMGB1 - an endogenous danger signal released from pyroptotic cells-in circulation, suggesting normal cell death induction in vivo. Moreover, their 
a
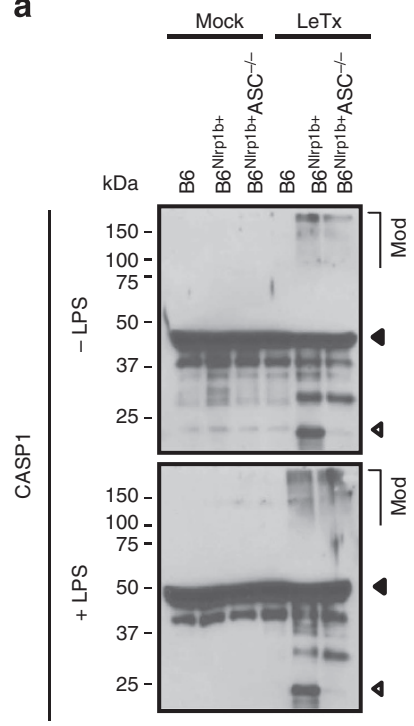

e

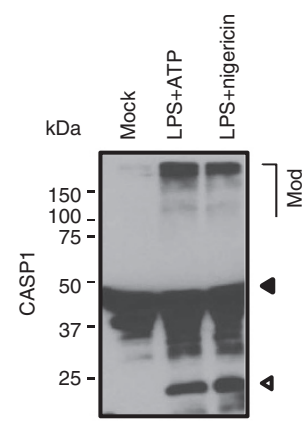

b
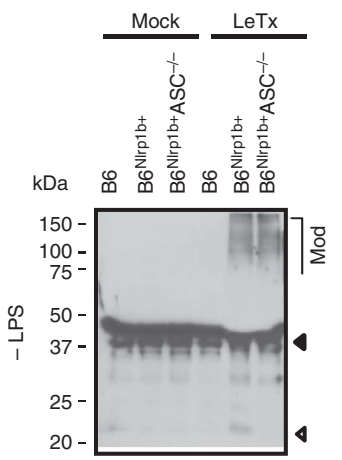

$\frac{5}{5}$

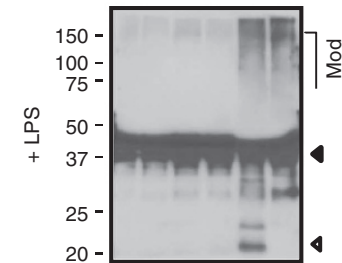

f

g c

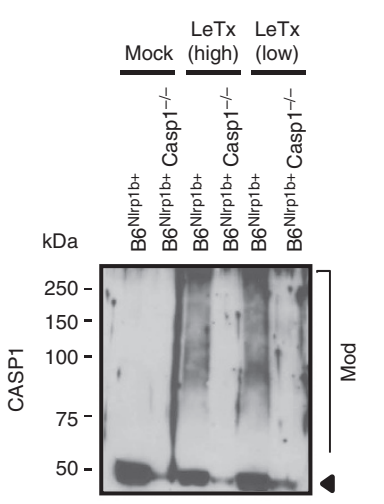

d

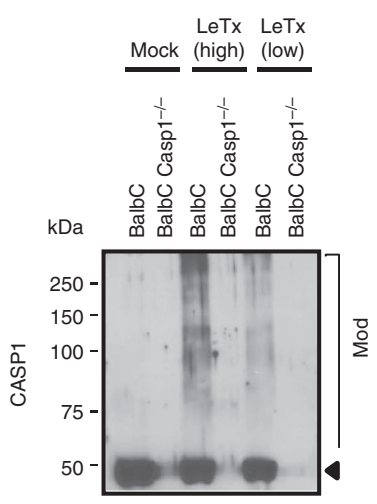

h
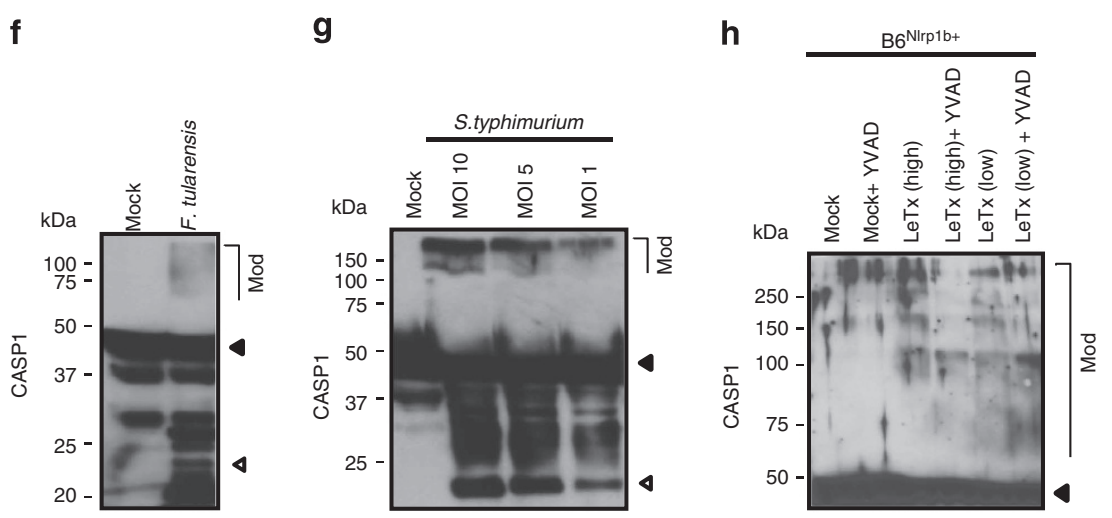

$\mathbf{j}_{\text {Time LeTx: }}$

$\mathbf{k}$

i
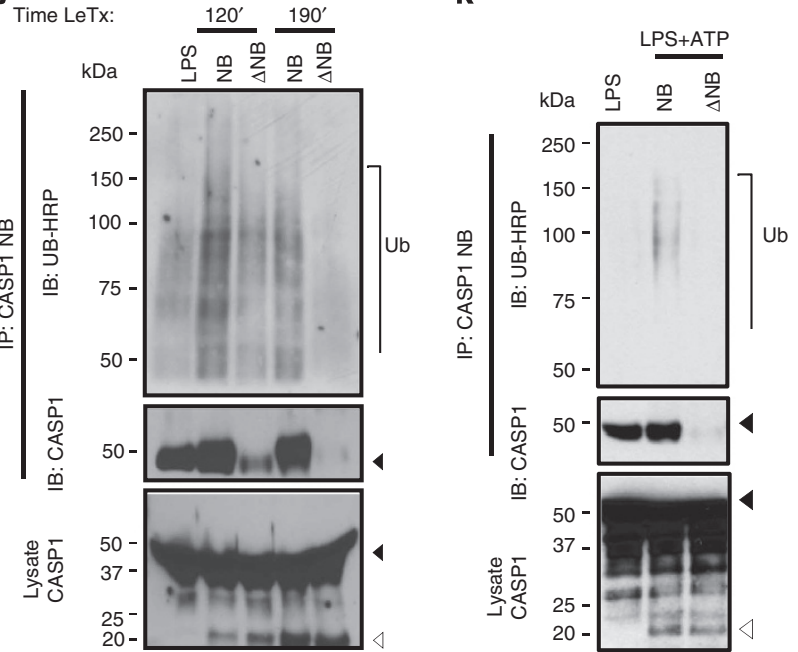

Figure 6 | ASC is dispensable for post-translational modification of active caspase-1. (a,b) B6, B6 $6^{\mathrm{Nlrp} 1 b+}$ and $\mathrm{B}^{\mathrm{Nlrp} 1 \mathrm{~b}+}+\mathrm{ASC}-/-$ BMDMs were left untreated or pretreated with $5 \mu \mathrm{g} \mathrm{ml}^{-1} \mathrm{LPS}$ for $3 \mathrm{~h}$ prior to being exposed to different concentrations of LeTx (a, $10 \mu \mathrm{g}$ PA $+10 \mu \mathrm{g}$ LF) or (b, $500 \mathrm{ng}$ $\mathrm{PA}+250 \mathrm{ng}$ LF) for another $3 \mathrm{~h}$. Cell lysates were immunoblotted for caspase-1. (c,d) B6 $6^{\mathrm{N} / r p 1 b}+$ and B6 $6^{\mathrm{N}} \mathrm{rp1b}+\mathrm{Casp1}-/-(\mathbf{c})$, or BALB/c and BALB/c Casp1 ${ }^{-/-}$BMDMs (d) were treated with $5 \mu \mathrm{g} \mathrm{ml}{ }^{-1}$ LPS for $3 \mathrm{~h}$ and subsequently exposed to $10 \mu \mathrm{g}$ PA $+10 \mu \mathrm{Lg}$ LF (high) or $500 \mathrm{ng}$ PA $+250 \mathrm{ng}$ LF (low) for another $3 \mathrm{~h}$. Cell lysates were immunoblotted for caspase-1. (e-g) Lysates of B6 BMDMs were immunoblotted for caspase-1 after cells have been primed with $5 \mu \mathrm{g} \mathrm{ml}^{-1} \mathrm{LPS}$ for $3 \mathrm{~h}$ and subsequently stimulated with $5 \mathrm{mM}$ ATP or $20 \mu \mathrm{M}$ nigericin for $30 \mathrm{~min}$ (e), infected with F. tularensis (m.o.i. 30 ) for $3 \mathrm{~h}$ (f), or infected with S. typhimurium (m.o.i. 10, 5 and 1) for $3 \mathrm{~h}$ (g). (h,i) LPS-primed B6 $6^{\mathrm{Nlp} p 1 b+}$ (h) and B6 ${ }^{\mathrm{Nlrp} 1 \mathrm{~b}+} \mathrm{ASC}^{-/-}$(i) BMDMs were pretreated with $50 \mu \mathrm{M}$ Ac-YVAD-cmk for 30 min prior to being exposed to $10 \mu \mathrm{g}$ PA $+10 \mu \mathrm{g}$ LF (High), or $500 \mathrm{ng}$ PA $+250 \mathrm{ng}$ LF (Low) for another $3 \mathrm{~h}$. Cell lysates were immunoblotted for caspase-1. (j,k) LPS-primed BALB/c BMDMs were exposed to $500 \mathrm{ng}$ PA $+250 \mathrm{ng} \mathrm{LF} \mathrm{ml}^{-1}$ for 120 or $190 \mathrm{~min}$, respectively (j), or treated with $5 \mathrm{mM}$ ATP for $30 \mathrm{~min}$ (k) before caspase-1 was immunoprecipitated using caspase-1 nanobody clone R2S40. Immunoprecipitates and lysates were immunoblotted for the indicated proteins. Data are representative of results from at least three independent experiments. 
a

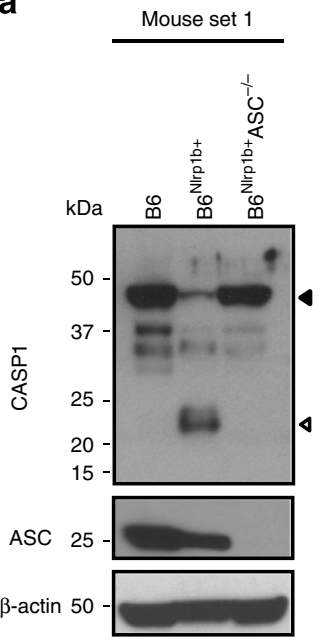

C

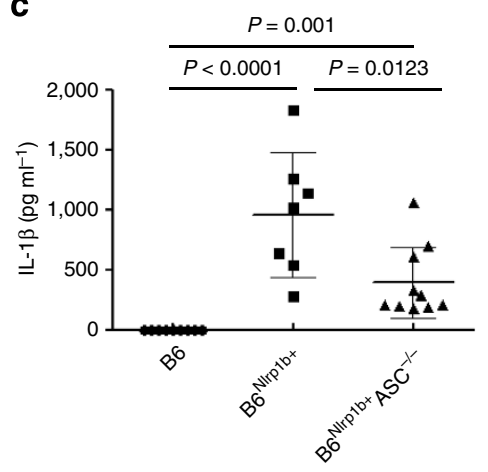

b

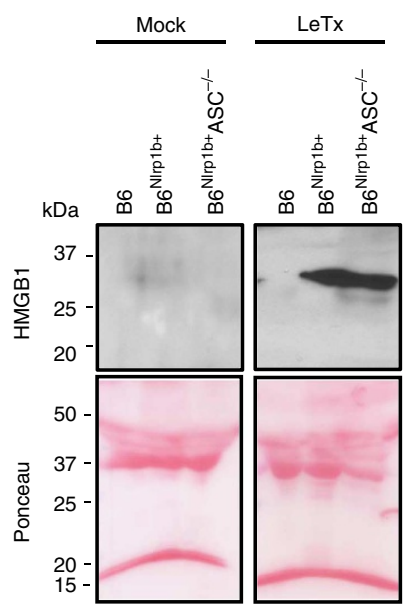

e

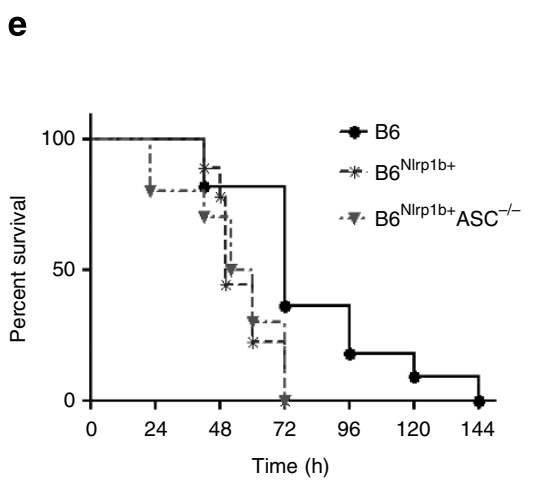

$\mathbf{f}$

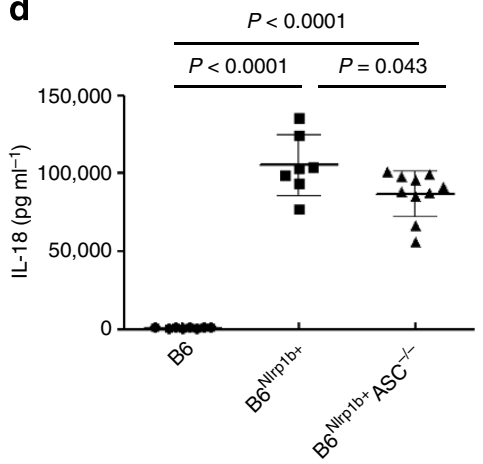

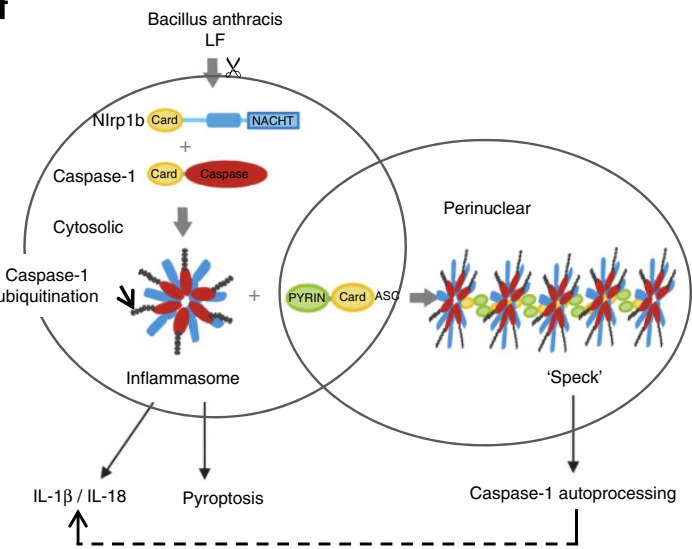

Figure 7 | In vivo role of ASC in LeTx-challenged mice. $(\mathbf{a}-\mathbf{d}) \mathrm{B} 6(n=9), \mathrm{B}^{\mathrm{Nlrp} 1 \mathrm{~b}}+(n=7)$ and $\mathrm{B}^{\mathrm{Nlrp} 1 \mathrm{~b}+} \mathrm{ASC}^{-/-}$mice $(n=10)$ were injected intraperitoneally with LeTx $(100 \mu \mathrm{g}$ PA and LF, respectively), and spleen and serum were collected $3 \mathrm{~h}$ later. Spleen (a) and serum samples (b) were immunoblotted for the indicated proteins. Ponceau staining was performed as loading control for serum samples (b). Circulating IL-1 13 (c) and IL-18 (d) levels in serum was determined by ELISA, with each dot representing an individual mouse. Statistical significance was determined by Student's $t$-test and P-values are indicated. (e) B6 $(n=11), \mathrm{B}^{\mathrm{Nlrp} 1 \mathrm{~b}}+(n=9)$ and $\mathrm{B}^{\mathrm{Nlrp} 1 \mathrm{~b}}+\mathrm{ASC}^{-/}-(n=10)$ mice were injected intraperitoneally with a lethal dose of LeTx (200 $\mu \mathrm{g}$ PA and LF, respectively) and survival was monitored over time. Statistical significance was determined by Kaplan-Meier analysis and $P$-values were as follows: $\mathrm{B} 6$ vs B6 ${ }^{\mathrm{Nlrp} 1 \mathrm{~b}+}, P=0.0096 ; \mathrm{B} 6$ vs B6 $6^{\mathrm{Nlrp} 1 \mathrm{~b}+} \mathrm{ASC}^{-/-}, P=0.0086 ; \mathrm{B}^{\mathrm{Nlrp} 1 \mathrm{~b}+}$ vs $\mathrm{B}^{\mathrm{Nlrp} 1 \mathrm{~b}+} \mathrm{ASC}-/-, P=0.79$ (non-significant). (f) Schematic model depicting the putative role of ASC in Nlrp1b inflammasome signalling in LeTx-challenged macrophages and mice.

serum contained significant amounts of IL-1 $\beta$ and IL-18, although the levels were detectably lower than in littermates expressing ASC. In contrast, mice lacking a functional NLRP1b allele were fully defective in both HMGB1 release and secretion of IL-1 $\beta$ and IL-18. As a result, ASC deletion failed to protect against LeTx-induced mortality, whereas the absence of a functional NLRP1b allele significantly delayed lethality. In conclusion, we showed that ASC is critical for LeTx-induced caspase-1 autoproteolysis and speck formation, but that these events are dispensable for NLRP1b inflammasome-mediated caspase- 1 activation, cytokine secretion and cell death induction in susceptible macrophages and in toxin-intoxicated mice. 


\section{Methods}

Mice. $B 6^{\mathrm{Nl} p 1 b+} A S C^{-1-}$ and $B 6^{\mathrm{Nlp} p 1 b+} \mathrm{Casp1^{-1- }}$ mice and littermate controls used in this study were obtained by breeding $B 6^{N l r p 1 b+}$ (ref. 8) to Asc ${ }^{-1-}$ (ref. 18) and $\mathrm{Casp1}^{-1-}$ (ref. 34) mice in a C57BL/6 genetic background, respectively. $\mathrm{Asc}^{-1-}$ and $\mathrm{Casp1}^{-1-}$ mice were also backcrossed to a $\mathrm{BALB} / \mathrm{c}$ genetic background for 10 generations. C57BL/6J, 129SVEV57 and BALB/c mice were originally purchased from Charles River and bred in-house. Animals were housed in individually ventilated cages under specific pathogen-free conditions, and studies were conducted under protocols approved by Ghent University and St Jude Committees on Use and Care of Animals.

Macrophage differentiation and stimulation. BMDMs were generated by culturing mouse bone marrow cells in L-cell-conditioned IMDM supplemented with $10 \% \mathrm{FBS}, 1 \%$ non-essential amino acid and $1 \%$ penicillin-streptomycin for 6 days. BMDMs were seeded in 12-well plates, and the next day either left untreated or stimulated with LPS $\left(5 \mu \mathrm{g} \mathrm{ml}^{-1}\right)$ for $3 \mathrm{~h}$ prior to treatment with different concentrations of anthrax PA, and wild-type or proteolytically inactive LF (Quadratech). In some experiments, BMDMs were pretreated with the caspase-1 inhibitor Ac-YVAD-cmk ( $50 \mu \mathrm{M}$; Enzo Life Sciences) for $30 \mathrm{~min}$ prior to LeTx incubation. Alternatively, macrophages were primed with LPS for $3 \mathrm{~h}$ and then stimulated with $20 \mu \mathrm{M}$ nigericin for $1 \mathrm{~h}$ or $5 \mathrm{mM}$ ATP for $30 \mathrm{~min}$. In other experiments, BMDMs were infected with S. typhimurium at m.o.i. of 10,5 or 1 for $3 \mathrm{~h}$ in a $\mathrm{CO}_{2}$ incubator at $37^{\circ} \mathrm{C}$, the last hour of which in the presence of gentamycin $\left(50 \mu \mathrm{g} \mathrm{ml}^{-1}\right)$. Alternatively, BMDMs were infected with $F$. tularensis at m.o.i. 30 for $3 \mathrm{~h}$, the last hour of which in the presence of gentamycin $\left(50 \mu \mathrm{g} \mathrm{ml}^{-1}\right)$. Finally, cytosolic dsDNA delivery was performed by transfecting cells for $24 \mathrm{~h}$ with Lipofectamine-2000 reagent (Life Technologies) according to the manufacturer's instructions.

Western blotting. Cell lysates, serum samples and spleen homogenates denatured in loading buffer containing SDS and $100 \mathrm{mM}$ DTT were boiled for $10 \mathrm{~min}$ before SDS-PAGE-separated proteins were transferred to PVDF membranes. Immunoblotting with primary antibodies against caspase-1 $(1 / 3,000$, kind gift of Dr Peter Vandenabeele, Ghent University), ASC (1/1,000, AG-25B-0006, Adipogen), IL-1 $\beta$ (1/2,000, GTX74034, GeneTex), caspase-11 (1/1,000, NB120-10454, Novus Biologicals), Ub-HRP (1/1,000, sc-8017, Santa Cruz), HMGB1 (1/100, 18256, Abcam), and $\beta$-actin $(1 / 20,000, \mathrm{NB} 600-501 \mathrm{H}$, Novus Biologicals) was followed by secondary anti-rabbit and anti-rat HRP antibodies $(1 / 5,000,111-035-144$ and 112-035-143, Jackson Immunoresearch). Ponceau S Solution was purchased from Sigma-Aldrich.

Fluorescence and confocal microscopy. B6, B6 $6^{N l p l b+}$ and $B^{N l p l p l b+} A S C^{-/-}$ macrophages grown on coverslips were either left untreated (mock) or stimulated with LeTx for $30 \mathrm{~min}$ after which the active caspase-1 FAM-YVAD-cmk FLICA probe (Gentaur Molecular Products) was added for another $45 \mathrm{~min}$. Cells were fixed and mounted on glass slides using ProLong Gold Antifade reagent containing DAPI (Life Technologies). Fluorescence micrographs were taken on an Olympus microscope using a $\times 20$ objective lens. Quantification of specks was performed in three micrographs and the mean grey value was determined in 10 cells using ImageJ software. Alternatively, cells grown on coverslips were treated with LeTx for $60 \mathrm{~min}$, fixed in $4 \%$ paraformaldehyde and then stained with antibodies against ASC (1/400, AG-25B-0006, Adipogen) and caspase-1 (1/200, AG-20B-0042-C100, Adipogen). Secundary antibodies anti-mouse Alexa Fluor 488 and anti-rabbit Alexa Fluor 594 (1/250, A-11029 and A-11037, Invitrogen) were used. Slides were mounted in ProLong Gold Antifade reagent with Dapi (P36935, Invitrogen). Confocal micrographs were taken on an Olympus microscope using a $\times 60$ objective lens. Quantification of specks was performed on 10 micrographs.

Immunoprecipitation. Treated BMDMs $\left(12 \times 10^{6}\right.$ cells per $10 \mathrm{~cm}$ dish) were lysed in $500 \mu \mathrm{l}$ Cell Lysis buffer $(10 \mathrm{mM}$ Tris- $\mathrm{HCl}$, pH $8,0.15 \mathrm{M} \mathrm{NaCl}, 2 \%$ SDS $)$ supplemented with $100 \mathrm{mM} \mathrm{N}$-ethylmaleimide (Sigma) and a Complete Protease Inhibitor Cocktail tablet (Roche Applied science) and supernatants were collected. Lysates and supernatants were boiled for $10 \mathrm{~min}$ followed by sonication for $5 \mathrm{~min}$. After centrifugation, supernatants and lysates were pooled and incubated with caspase-1-specific nanobody clone R2S40 that was developed in-house $(1 / 100$, VIB Nanobody Service Facility) on a rotating platform at $4{ }^{\circ} \mathrm{C}$ for $1 \mathrm{~h}$. Subsequently, HA-beads were added and incubated overnight on a rotating platform at $4{ }^{\circ} \mathrm{C}$. The next day, beads were washed three times before being resuspended in $1 \times$ Laemmli buffer and boiled for $10 \mathrm{~min}$. Eluted samples were analysed by SDS-PAGE

In vivo LeTx challenge. Cohorts of 8-12 week-old male and female B6, $B 6^{N l r p 1 b+}$ and $B 6^{N l r p 1 b+} A S C^{-1-}$ littermates were injected intraperitoneally with $100 \mu \mathrm{g}$ $\mathrm{PA}+100 \mu \mathrm{g}$ LF for $3 \mathrm{~h}$ before serum was collected for cytokine analysis, and spleen homogenates were prepared for immunoblotting. In other experiments, groups of $\mathrm{B} 6, B 6^{\mathrm{Nl} p l b+}$ and $B 6^{\mathrm{Nl} p 1 b+} A S C^{-1-}$ mice were injected intraperitoneally with $200 \mu \mathrm{g}$ PA $+200 \mu \mathrm{g}$ LF to monitor survival.
Cytokine analysis. Cytokine levels in cell culture medium and serum were determined by multiplex ELISA (Bio-Rad), IL1 $\beta$ ELISA (R\&D Systems) and IL-18 ELISA (MBL international), according to the manufacturers' instructions.

LDH measurement. Cell death levels were determined by LDH assay in culture medium according to the manufacturer's instructions (Promega).

RT-qPCR. Total RNA was isolated using the Qiashredder and RNeasy Mini kits (Qiagen), according to the manufacturer's instructions. RNA ( $1 \mu \mathrm{g})$ was used for cDNA synthesis with iScript reagents (Bio-Rad). Quantitative PCR on duplicate samples was performed using the LightCycler 480 SYBR Green master mix (Roche) and normalized to the housekeeping gene $\beta$-actin. Primers used were $5^{\prime}$-CAACAA GACTTGAACACAACGAG- $3^{\prime}$ and $5^{\prime}$-CTCTCAATGACTGTGCTGGGTA-3' for NLRP $1 b^{8}$; and $5^{\prime}$-GTCGACAACGGCTCCGGC-3' and $5^{\prime}$-GGTGTGGT GCCAGATTTTCT- $3^{\prime}$ for $\beta$-actin.

Statistics. GraphPad Prism 5.0 software was used for data analysis. Data are represented with s.d. or s.e.m., as indicated. Statistical significance was determined by Student's $t$-test for cell death and cytokine assays, and Kaplan-Meier analysis was used to analyse mortality; $P<0.05$ was considered statistically significant.

\section{References}

1. Lamkanfi, M. \& Dixit, V. M. Inflammasomes and their roles in health and disease. Annu. Rev. Cell Dev. Biol. 28, 137-161 (2012).

2. Miao, E. A. et al. Caspase-1-induced pyroptosis is an innate immune effector mechanism against intracellular bacteria. Nat. Immunol. 11, 1136-1142 (2010)

3. Lamkanfi, M., Walle, L. V. \& Kanneganti, T. D. Deregulated inflammasome signaling in disease. Immunol. Rev. 243, 163-173 (2011).

4. Martinon, F., Burns, K. \& Tschopp, J. The inflammasome: a molecular platform triggering activation of inflammatory caspases and processing of proIL-beta. Mol. Cell 10, 417-426 (2002).

5. Lamkanfi, M., Declercq, W., Kalai, M., Saelens, X. \& Vandenabeele, P. Alice in caspase land. A phylogenetic analysis of caspases from worm to man. Cell Death Differ. 9, 358-361 (2002).

6. Warren, S. E. et al. Cutting edge: cytosolic bacterial DNA activates the inflammasome via Aim2. J. Immunol. 185, 818-821 (2010).

7. Rathinam, V. A. et al. The AIM2 inflammasome is essential for host defense against cytosolic bacteria and DNA viruses. Nat. Immunol. 11, 395-402 (2010).

8. Boyden, E. D. \& Dietrich, W. F. Nalp1b controls mouse macrophage susceptibility to anthrax lethal toxin. Nat. Genet. 38, 240-244 (2006).

9. Collier, R. J. \& Young, J. A. Anthrax toxin. Annu. Rev. Cell Dev. Biol. 19, 45-70 (2003).

10. Fink, S. L., Bergsbaken, T. \& Cookson, B. T. Anthrax lethal toxin and Salmonella elicit the common cell death pathway of caspase-1-dependent pyroptosis via distinct mechanisms. Proc. Natl Acad. Sci. USA 105, 4312-4317 (2008).

11. Frew, B. C., Joag, V. R. \& Mogridge, J. Proteolytic processing of Nlrplb is required for inflammasome activity. PLoS Pathog. 8, e1002659 (2012)

12. Liao, K. C. \& Mogridge, J. Activation of the Nlrplb inflammasome by reduction of cytosolic ATP. Infect. Immun. 81, 570-579 (2013).

13. Nour, A. M. et al. Anthrax lethal toxin triggers the formation of a membraneassociated inflammasome complex in murine macrophages. Infect. Immun. 77, 1262-1271 (2009).

14. de Rivero Vaccari, J. P. et al. Therapeutic neutralization of the NLRP1 inflammasome reduces the innate immune response and improves histopathology after traumatic brain injury. J. Cereb. Blood Flow. Metab. 29, 1251-1261 (2009).

15. Kanneganti, T. D. et al. Critical role for Cryopyrin/Nalp3 in activation of caspase-1 in response to viral infection and double-stranded RNA. J. Biol. Chem. 281, 36560-36568 (2006).

16. Hornung, V. et al. AIM2 recognizes cytosolic dsDNA and forms a caspase-1activating inflammasome with ASC. Nature 458, 514-518 (2009).

17. Broz, P., von Moltke, J., Jones, J. W., Vance, R. E. \& Monack, D. M. Differential requirement for Caspase-1 autoproteolysis in pathogen-induced cell death and cytokine processing. Cell Host Microbe 8, 471-483 (2010).

18. Mariathasan, S. et al. Differential activation of the inflammasome by caspase-1 adaptors ASC and Ipaf. Nature 430, 213-218 (2004).

19. Kanneganti, T. D. et al. Bacterial RNA and small antiviral compounds activate caspase-1 through cryopyrin/Nalp3. Nature 440, 233-236 (2006).

20. Lamkanfi, M. Emerging inflammasome effector mechanisms. Nat. Rev. Immunol. 11, 213-220 (2011).

21. Kayagaki, N. et al. Non-canonical inflammasome activation targets caspase-11. Nature 479, 117-121 (2011).

22. Huang, M. T. et al. Critical role of apoptotic speck protein containing a caspase recruitment domain (ASC) and NLRP3 in causing necrosis and ASC speck formation induced by Porphyromonas gingivalis in human cells. J. Immunol. 182, 2395-2404 (2009).

23. Jones, J. W. et al. Absent in melanoma 2 is required for innate immune recognition of Francisella tularensis. Proc. Natl Acad. Sci. USA 107, 9771-9776 (2010). 
24. Newman, Z. L. et al. Susceptibility to anthrax lethal toxin-induced rat death is controlled by a single chromosome 10 locus that includes rNlrp1. PLoS Pathog. 6, e1000906 (2010).

25. Moayeri, M., Martinez, N. W., Wiggins, J., Young, H. A. \& Leppla, S. H. Mouse susceptibility to anthrax lethal toxin is influenced by genetic factors in addition to those controlling macrophage sensitivity. Infect. Immun. 72, 4439-4447 (2004).

26. Friedlander, A. M. Macrophages are sensitive to anthrax lethal toxin through an acid-dependent process. J. Biol. Chem. 261, 7123-7126 (1986).

27. Hanna, P. C., Acosta, D. \& Collier, R. J. On the role of macrophages in anthrax. Proc. Natl Acad. Sci. USA 90, 10198-10201 (1993).

28. Welkos, S. L., Keener, T. J. \& Gibbs, P. H. Differences in susceptibility of inbred mice to Bacillus anthracis. Infect. Immun. 51, 795-800 (1986)

29. Hanna, P. How anthrax kills. Science 280, 1673-1674 (1998).

30. Duesbery, N. S. et al. Proteolytic inactivation of MAP-kinase-kinase by anthrax lethal factor. Science 280, 734-737 (1998).

31. Faustin, B. et al. Reconstituted NALP1 inflammasome reveals two-step mechanism of caspase-1 activation. Mol. Cell 25, 713-724 (2007).

32. Boatright, K. M. \& Salvesen, G. S. Mechanisms of caspase activation. Curr. Opin. Cell Biol. 15, 725-731 (2003).

33. Salvesen, G. S. \& Dixit, V. M. Caspase activation: the induced-proximity model. Proc. Natl Acad. Sci. USA 96, 10964-10967 (1999).

34. Kuida, K. et al. Altered cytokine export and apoptosis in mice deficient in interleukin-1 beta converting enzyme. Science 267, 2000-2003 (1995).

\section{Acknowledgements}

We thank Dr Vishva Dixit (Genentech), Dr Eric D. Boyden and Dr William F Dietrich (Harvard) and Dr Richard Flavell (Yale) for generous supply of mutant mice. Caspase-1 antiserum for western blotting was a kind gift of Dr Peter Vandenabeele (VIB/Ghen University). L.V.W. is a postdoctoral fellow with the Fund for Scientific ResearchFlanders. T.-D.K. is supported by grants from the National Institute of Health (Grants AR056296, CA163507 and AI101935) and the American Lebanese Syrian Associated Charities (ALSAC). This work was supported in part by grants from the European Research Council (Grant 281600) and the Fund for Scientific Research-Flanders (grants G030212N, 1.2.201.10.N.00 and 1.5.122.11.N.00) to M.L.

\section{Author contributions}

N.V.O., T.-D.K. and M.L. designed the study; N.V.O., P.G., L.V.W. and A.F. performed experiments; N.V.O., T.-D.K. and M.L. analysed the data and wrote the manuscript; M.L. oversaw the project.

\section{Additional information}

Supplementary Information accompanies this paper at http://www.nature.com/ naturecommunications

Competing financial interests: The authors declare no competing financial interests.

Reprints and permission information is available online at http://npg.nature.com/ reprintsandpermissions/

How to cite this article: Van Opdenbosch, N. et al. Activation of the NLRP1b inflammasome independently of ASC-mediated caspase-1 autoproteolysis and speck formation. Nat. Commun. 5:3209 doi: 10.1038/ncomms4209 (2014).

(c) (1) $\ominus$ This work is licensed under a Creative Commons Attributioncc) NonCommercial-NoDerivs 3.0 Unported License. To view a copy of this license, visit http://creativecommons.org/licenses/by-nc-nd/3.0/ 